\title{
Distributed Space-Time Coding for Two-Way Wireless Relay Networks
}

\author{
Tao Cui, Student Member, IEEE, Feifei Gao, Member, IEEE, Tracey Ho, Member, IEEE, and \\ Arumugam Nallanathan, Senior Member, IEEE
}

\begin{abstract}
In this paper, we consider distributed space-time coding for two-way wireless relay networks, where communication between two terminals is assisted by relay nodes. Relaying protocols using two, three, and four time slots are proposed. The protocols using four time slots are the traditional amplify-and-forward (AF) and decode-and-forward (DF) protocols, which do not consider the property of the two-way traffic. A new class of relaying protocols, termed as partial decode-and-forward (PDF), is developed for the two time slots transmission, where each relay first removes part of the noise before sending the signal to the two terminals. Protocols using three time slots are proposed to compensate the fact that the two time slots protocols cannot make use of direct transmission between the two terminals. For all protocols, after processing their received signals, the relays encode the resulting signals using a distributed linear dispersion (LD) code. The proposed AF protocols are shown to achieve the diversity order of $\min \{N, K\}(1-(\log \log P / \log P))$, where $N$ is the number of relays, $P$ is the total power of the network, and $K$ is the number of symbols transmitted during each time slot. When random unitary matrix is used for $L D$ code, the proposed PDF protocols resemble random linear network coding, where the former operates on the unitary group and the latter works on the finite field. Moreover, PDF achieves the diversity order of $\min \{N, K\}$ but the conventional DF can only achieve the diversity order of 1 . Finally, we find that two time slots protocols also have advantages over four-time-slot protocols in media access control (MAC) layer.
\end{abstract}

Index Terms-Rayleigh-fading channels, space-time coding, two-way channel, wireless relay networks.

\section{INTRODUCTION}

$\mathbf{S}$ EVERAL works on wireless networks consider the exploitation of spatial diversity using antennas of different users in the network [1]-[4]. In [1], spatial diversity was exploited by extending the existing strategies, e.g., amplify-and-forward (AF) and decode-and-forward (DF), from

Manuscript received May 03, 2008; revised October 12, 2008. First published November 07, 2008; current version published January 30, 2009. The associate editor coordinating the review of this manuscript and approving it for publication was Dr. Subhrakanti Dey. This work has been supported in part by DARPA Grant N66001-06-C-2020, Caltech's Lee Center for Advanced Networking, the Okawa Foundation Research Grant, and a gift from Microsoft Research, and the National University of Singapore and Defence Science and Technology Agency (DSTA), Singapore, under Grant R-263-000-447-232/123. This paper has been presented in part at the IEEE International Conference on Communications, Beijing, China, May 2008.

T. Cui and T. Ho are with the Department of Electrical Engineering, California Institute of Technology, Pasadena, CA 91125 USA (e-mail: taocui @ caltech.edu; tho@caltech.edu).

F. Gao is with the Institute for Infocomm Research, A*STAR, 138632, Singapore (e-mail: feifeigao@ieee.org).

A. Nallanathan is with the Division of Engineering, King's College London, London WC2R 2LS, U.K. (e-mail: arumugam.nallanathan@kcl.ac.uk).

Digital Object Identifier 10.1109/TSP.2008.2009025 one-way relay channels [5]. In [2], a distributed linear dispersion (LD) space-time code was proposed using the AF protocol, where both the diversity gain and coding gain are analyzed. Distributed space-time block coding for DF protocols was proposed in [3] and its randomized version was given in [4]. These works [1]-[4] consider only unidirectional communication.

Two-way communication is another common communication scenario where two parties transmit information to each other. The two-way channel was first considered by Shannon [6], who derived inner and outer bounds on the capacity region. Recently, considering the two-way channel over relay networks (TWRC) has drawn renewed interest from both academic and industrial communities [7]-[9] due to its potential application to enable range-rate enhancements of future cellular systems. By considering TWRC as a component of a general wireless network, TWRC could also lead to network resources saving in multi-hop networks such as sensor networks and ad hoc networks. In [7], both $\mathrm{AF}$ and $\mathrm{DF}$ protocols from one-way relay channels were extended to the half-duplex Gaussian TWRC. In [9], algebraic network coding [10], [11] was used to increase the sum-rate of two users. By network coding, each node is allowed to perform algebraic operations on received packets instead of only forwarding or replicating them.

In this paper, we design distributed space-time coding (DSTC) for two-way wireless relay networks with fading channels. Relaying protocols using two, three, and four time slots are proposed. In the two-time-slot protocols, the terminals transmit simultaneously during the first time slot. In the three-time-slot protocols, the terminals transmit separately during the first two time slots, and the relays transmit in the third time slot by combining its received signals in the first two time slots. In the four-time-slot protocols, one terminal transmits during the first time slot and the relays transmit during the second time slot, while the other terminal transmits during the third time slot and the relays transmit during the fourth time slot.

For two-time-slot transmission, a class of relaying protocols, termed as partial decode-and-forward (PDF), is developed. Specifically, we propose two PDF protocols, denoted as PDF I and PDF II, under which each relay removes part of the noise before sending the signal to two terminals. Both AF and PDF I actually transmit the sum of the signals from two terminals in the complex field. However, PDF II, inspired by network coding, conceals information by performing a modular operation on the denoised signal or operating on a modular group. Since two-time-slot protocols cannot make use of the direct link between the two terminals, we then consider protocols using three time slots. For all two, three, and four-time-slot 


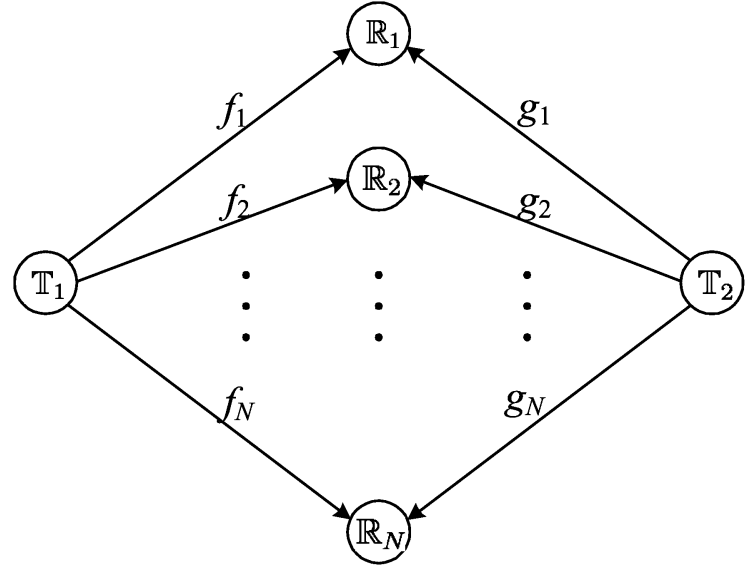

(a) Uplink Channel

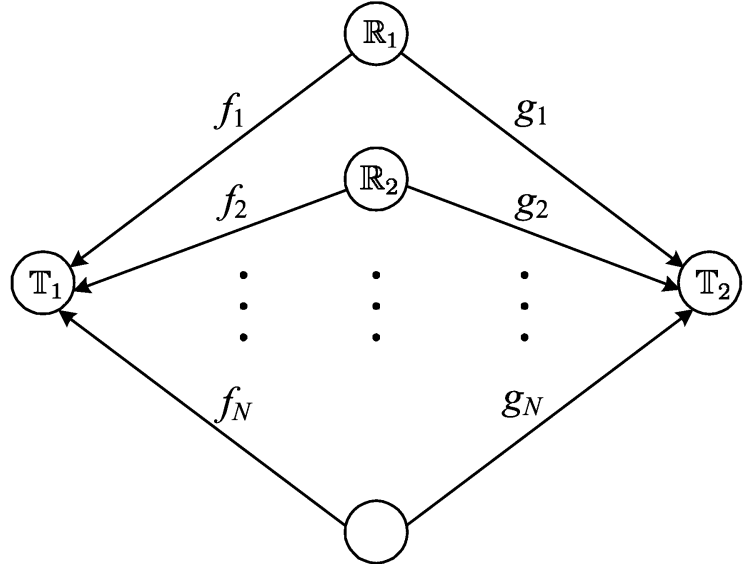

(b) Downlink Channel

Fig. 1. Two-way relay wireless network: (a) uplink channel from the terminals to the relays and (b) downlink channel from the relays to the terminals.

protocols, the relays encode using a distributed linear dispersion (LD) code [12]. From the analytical studies, we show that the proposed AF protocols can achieve the diversity order of $\min \{N, K\}(1-(\log \log P / \log P))$. Moreover, with the aid of cyclic redundancy check (CRC), PDF II can achieve a diversity order of $\min \{N, K\}$. It should be mentioned that the achievable diversity order is derived by using union bound rather than through a precise performance analysis. When random unitary matrix is used as LD code, the distributed operation of the network can be achieved without explicit code construction for a specific network. The effect of different protocols on the medium access control (MAC) layer is also investigated. Assuming slotted ALOHA is used for MAC, we find that the two-time-slot protocol reduces the number of transmissions per information symbol by $50 \%$ compared to the four-time-slot protocols. Our simulation results support the analysis, from which we see that PDF I performs better than AF while PDF II yields the best performance. The results in this paper show that by carefully designing protocols, resources saving could be realized by using TWRC as a basic component in larger wireless networks.

The rest of the paper is organized as follows. In Section II, we present the TWRC model. We then develop the different space time coding protocols, assuming, four-, two-, and three-timeslot transmissions, in Section III, Section IV, and Section V, respectively. The performance analysis of some representative protocols are provided in Section VI. In Section VII, the numerical results are provided to corroborate our proposed studies. Finally, conclusions are given in Section VIII.

Notations: Vectors and matrices are denoted using boldface small and capital letters, respectively; the transpose, complex conjugate, Hermitian, and inverse of the matrix $\mathbf{A}$ are denoted by $\mathbf{A}^{T}, \mathbf{A}^{*}, \mathbf{A}^{H}$, and $\mathbf{A}^{-1}$, respectively; $\|\mathbf{a}\|_{2}$ is the $l_{2}$ norm of a; $\operatorname{diag}\{\mathbf{a}\}$ denotes a diagonal matrix with the diagonal element constructed from $\mathbf{a} ; \mathbf{I}_{K}$ is the $K \times K$ identity matrix; $E\{\cdot\}$ denotes the statistical expectation. The AF protocol using $t$ time slots is denoted by $t$-AF.

\section{NETWORK MODEL}

We consider a wireless network with $N$ relay nodes $\mathbb{R}_{i}, i=$ $1, \ldots, N$, and two terminal nodes $\mathbb{T}_{m}, m=1,2$ as shown in Fig. 1. The two terminals exchange information with the assistance of relays in between. Every node has only a single antenna that cannot transmit and receive simultaneously. For simplicity, we assume that the uplink channel from the terminals to the relays and the downlink channel from the relays to the terminals are reciprocal, i.e., the uplink channel gain from $\mathbb{T}_{m}$ to $\mathbb{R}_{i}$ is identical to the downlink channel gain from $\mathbb{R}_{i}$ to $\mathbb{T}_{m}$. Denote the channel between $\mathbb{T}_{1}$ and $\mathbb{R}_{i}$ by $f_{i}$, and the channel between $\mathbb{T}_{2}$ and $\mathbb{R}_{i}$ by $g_{i}$. We assume that $f_{i}$ and $g_{i}$ are independent complex Gaussian random variables with zero mean and unit variance, i.e., $f_{i} \sim \mathcal{C N}(0,1)$ and $g_{i} \sim \mathcal{C N}(0,1)$. Moreover, all channels are quasi-stationary which are constant within the number of time slots considered. Except in Section V, we assume there is no direct connection between the source and the destination as in [7] (for example due to shadowing or too large separation). We further assume symbol-level synchronization at the relays, which is less restrictive than packet-level synchronization. We also assume that the channel is unknown to the transmitting nodes but is perfectly known at the receiving nodes, which can be achieved by adapting the estimation algorithms in [13].

Assume that terminal $\mathbb{T}_{m}$ wishes to send the signal $\mathbf{s}_{m}=$ $\left[s_{m, 1}, \ldots, s_{m, K}\right]^{T}$ to the other terminal, where $s_{m, t} \in \mathcal{A}_{m}$, $m=1,2, t=1, \ldots, K, \mathcal{A}_{m}$ is a finite constellation with average power 1 , and $K$ is the length of each time slot. Thus, $E\left\{\mathbf{s}_{m}^{H} \mathbf{s}_{m}\right\}=K$. The average power of terminal $\mathbb{T}_{m}$ is denoted as $P_{m}, m=1,2$. For a fair comparison, we assume that the total power on all relays is a constant $P_{3}$ and each relay has power $P_{3} / N$ due to the symmetry. For convenience, the noise variance at $\mathbb{R}_{i}$ or $\mathbb{T}_{i}$ is assumed to be 1 .

When all relays do not transmit, the $\mathbb{R}_{i}$ receives at time $k$

$$
y_{r, i}[k]=f_{i}[k] x_{1}[k]+g_{i}[k] x_{2}[k]+n_{r, i}[k]
$$


where $x_{m}[k]$ is the transmitted signal by $\mathbb{T}_{m}$ at time $k$, and $n_{r, i}[k] \sim \mathcal{C} \mathcal{N}(0,1)$ is the additive white Gaussian noise at the $\mathbb{R}_{i}$. When both terminals choose to receive at time $k$, they get

$$
\begin{aligned}
& y_{1}[k]=\sum_{i=1}^{N} f_{i}[k] x_{r, i}[k]+n_{1}[k] \\
& y_{2}[k]=\sum_{i=1}^{N} g_{i}[k] x_{r, i}[k]+n_{2}[k]
\end{aligned}
$$

where $x_{r, i}[k]$ is the transmitted signal by $\mathbb{R}_{i}, i=1, \ldots, N$ at time $k$, and $n_{m}[k] \sim \mathcal{C N}(0,1)$ is the additive white Gaussian noise at $\mathbb{T}_{m}$. Due to the quasi-stationarity assumption on the channel gains, we remove the time index in $f_{i}$ and $g_{i}$ in the following.

\section{Distributed Space-Time Protocols USING FOUR TIME SLOTS}

The protocols in this section simply apply traditional space time protocols [1]-[4] separately for each direction of traffic. In the first time slot, $\mathbb{T}_{1}$ sends its data to all the relays. The relays transmit a function of their received signals to $\mathbb{T}_{2}$ in the second time slot. In the third time slot, $\mathbb{T}_{2}$ sends its data to the relays. The relays transmit transformed signals to $\mathbb{T}_{1}$ in the fourth time slot. We, therefore, have

$$
\begin{aligned}
{\left[x_{1}[1], \ldots, x_{1}[K]\right]^{T} } & =\alpha_{1}\left(\mathbf{A}_{1} \mathbf{s}_{1}+\mathbf{B}_{1} \mathbf{s}_{1}^{*}\right), \\
x_{1}[k] & =0, \quad k=K+1, \ldots, 4 K
\end{aligned}
$$

and

$$
\begin{aligned}
{\left[x_{2}[2 K+1], \ldots, x_{2}[3 K]\right]^{T} } & =\alpha_{2}\left(\mathbf{A}_{2} \mathbf{s}_{2}+\mathbf{B}_{2} \mathbf{s}_{2}^{*}\right) \\
x_{2}[k] & =0, \text { otherwise }
\end{aligned}
$$

where $\mathbf{A}_{i}$ and $\mathbf{B}_{i}$ are $K \times K$ precoding matrices, ${ }^{1} i=1,2$, and $\alpha_{i}$ is a scalar to satisfy average power constraint. To simplify analysis, we assume $\mathbf{A}_{i}$ is unitary and $\mathbf{B}_{i}=\mathbf{0}$. Due to the symmetry between the first two time slots and the last two time slots, we focus on the first two time slots in the following. From (1)-(4), we can simplify the signal model as

$$
\mathbf{y}_{r, i}=\sqrt{4 P_{1}} f_{i} \mathbf{A}_{1} \mathbf{s}_{1}+\mathbf{n}_{r, i}, \quad \mathbf{y}_{2}=\sum_{i=1}^{N} \beta_{i} g_{i} \mathbf{x}_{r, i}+\mathbf{n}_{2}
$$

where

$$
\begin{aligned}
\mathbf{y}_{r, i} & =\left[y_{r, i}[1], \ldots, y_{r, i}[K]\right]^{T} \\
\mathbf{y}_{2} & =\left[y_{2}[K+1], \ldots, y_{2}[2 K]\right]^{T} \\
\mathbf{x}_{r, i} & =\left[x_{r, i}[K+1], \ldots, x_{r, i}[2 K]\right]^{T} \\
\mathbf{n}_{r, i} & =\left[n_{r, i}[1], \ldots, n_{r, i}[K]\right]^{T} \\
\mathbf{n}_{2} & =\left[n_{2}[K+1], \ldots, n_{2}[2 K]\right]^{T}
\end{aligned}
$$

\footnotetext{
${ }^{1}$ Note that the precoding matrices are not necessarily square matrices, for example when less than $K$ symbols are sent by each terminal. We have assumed linear precoding in this paper. Nonlinear precoding can also be used.
}

and $\beta_{i}$ is a scalar to satisfy the power constraint at $\mathbb{R}_{i}$. The protocols described below differ in the way they form the transmitted signals $\mathbf{x}_{r, i}$ at relays.

\section{A. Amplify-and-Forward (4-AF)}

This protocol simply employs the one-way AF protocol of [2] for each direction of traffic. At each relay, $\mathbf{y}_{r, i}$ is first precoded by a unitary matrix $\mathbf{A}_{r, i}$ and is then scaled by a factor $\beta_{i}$ to satisfy the average power constraint as in [2]. There are two different choices of $\beta_{i}$ :

$$
\begin{aligned}
& \beta_{i}=\sqrt{\frac{2 P_{3}}{N\left(4\left|f_{i}\right|^{2} P_{1}+1\right)}} \\
& \beta_{i}=\sqrt{\frac{2 P_{3}}{N\left(4 P_{1}+1\right)}} .
\end{aligned}
$$

In this paper, we consider the $\beta_{i}$ in (6b) which makes the analysis tractable and does not require the relay to estimate the channel from terminal to the relay. With this choice of $\beta_{i}$, we can write the received signal as

$$
\mathbf{y}_{2}=\sqrt{\frac{8 P_{1} P_{3}}{N\left(4 P_{1}+1\right)}} \mathbf{S}_{1} \mathbf{h}+\mathbf{w}_{2}=\sqrt{\frac{8 P_{1} P_{3}}{N\left(4 P_{1}+1\right)}} \mathbf{H s}_{1}+\mathbf{w}_{2}
$$

where

$$
\begin{aligned}
\mathbf{S}_{1} & =\left[\mathbf{A}_{r, 1} \mathbf{A}_{1} \mathbf{s}_{1}, \ldots, \mathbf{A}_{r, N} \mathbf{A}_{1} \mathbf{s}_{1}\right] \\
\mathbf{h} & =\left[f_{1} g_{1}, \ldots, f_{N} g_{N}\right]^{T} \\
\mathbf{H} & =\sum_{i=1}^{N} f_{i} g_{i} \mathbf{A}_{r, i} \mathbf{A}_{1} \\
\mathbf{w}_{2} & =\sqrt{\frac{2 P_{3}}{N\left(4 P_{1}+1\right)} \sum_{i=1}^{N} g_{i} \mathbf{A}_{r, i} \mathbf{n}_{r, i}+\mathbf{n}_{2} .}
\end{aligned}
$$

The maximum-likelihood (ML) decoding of (7) at $\mathbb{T}_{2}$ is

$$
\hat{\mathbf{s}}_{1}=\underset{\tilde{\mathbf{s}}_{1} \in \mathcal{A}_{1}^{N}}{\operatorname{argmin}}\left\|\mathbf{y}_{2}-\sqrt{\frac{8 P_{1} P_{3}}{N\left(4 P_{1}+1\right)}} \mathbf{H} \tilde{\mathbf{s}}_{1}\right\|_{2}^{2}
$$

which can be solved efficiently by using sphere decoder [14] and its variants [15]. From (8), if we define $\tilde{\mathbf{A}}_{r, i}=\mathbf{A}_{r, i} \mathbf{A}_{1}$ as another unitary matrix, the system is unchanged. Therefore, in this protocol, we can simply set $\mathbf{A}_{1}=\mathbf{I}_{K}$ as in [2]. Note that this protocol has been analyzed in [2]. In all the AF protocols, we set $\mathbf{A}_{1}=\mathbf{A}_{2}=\mathbf{I}_{K}$ for simplicity.

\section{B. Decode-and-Forward}

In DF protocol, the $\mathbb{R}_{i}$ first decodes $\mathbf{s}_{1}$ via ML decoding as

$$
\hat{\mathbf{s}}_{1, i}=\underset{\tilde{\mathbf{s}}_{1} \in \mathcal{A}_{1}^{N}}{\operatorname{argmin}}\left\|\mathbf{y}_{r, i}-\sqrt{4 P_{1}} f_{i} \mathbf{A}_{1} \tilde{\mathbf{s}}_{1}\right\|_{2}^{2}
$$

which can also be solved by sphere decoder. At each relay, $\hat{\mathbf{s}}_{1, i}$ is first precoded according to

$$
\mathbf{x}_{r, i}=\mathbf{A}_{r, i} \hat{\mathbf{s}}_{1, i}+\mathbf{B}_{r, i} \hat{\mathbf{s}}_{1, i}^{*}
$$


and is then scaled by a factor $\beta_{i}$ to satisfy the average power constraint. The distributed space time block codes in [3] are special cases of (14), and [3] assumes perfect decoding at the relay, i.e., $\hat{\mathbf{s}}_{1, i}=\mathbf{s}_{1}$. The decoding at the end terminals is similar to (12). From simulation results, we observe that DF can only achieve a diversity order 1 with the nonperfect decoding at the relay.

\section{DSTC Protocols Using Two Time Slots}

In two-time-slot relaying, both $\mathbb{T}_{1}$ and $\mathbb{T}_{2}$ simultaneously send their data to all the relays in the first time slot. Since each terminal transmits every two time slots, the transmit power is $\sqrt{2 P_{i}}$ and $\mathbb{R}_{i}$ receives

$$
\mathbf{y}_{r, i}=\sqrt{2 P_{1}} f_{i} \mathbf{s}_{1}+\sqrt{2 P_{2}} g_{i} \mathbf{s}_{2}+\mathbf{n}_{r, i}
$$

where $\mathbf{n}_{r, i}$ is the $K \times 1$ vector representing the circularly complex Gaussian noise. In the second time slot, $\mathbb{R}_{i}$ transmits $\mathbf{x}_{r, i}$ (obtained as a function of $\mathbf{y}_{r, i}$ as described in the various protocols below) scaled by $\beta_{i}$ to maintain average power $P_{3}$. The received signals at the two terminals are then

$$
\mathbf{y}_{1}=\sum_{i=1}^{N} \beta_{i} f_{i} \mathbf{x}_{r, i}+\mathbf{n}_{1}, \quad \mathbf{y}_{2}=\sum_{i=1}^{N} \beta_{i} g_{i} \mathbf{x}_{r, i}+\mathbf{n}_{2}
$$

where $\mathbf{n}_{m}$ is the noise vector at $\mathbb{T}_{m}, m=1,2$.

\section{A. Amplify-and-Forward (2-AF)}

Like the 4-AF protocol in Section IV, $\mathbf{x}_{r, i}$ is obtained by precoding $\mathbf{y}_{r, i}$ with a unitary matrix $\mathbf{A}_{r, i}$, and is then scaled by $\beta \triangleq \beta_{i}=\sqrt{\left(2 P_{3} / N\left(2 P_{1}+2 P_{2}+1\right)\right)}$ to satisfy the average power constraint. Due to symmetry, we will only consider the received signal at $\mathbb{T}_{2}$, which is

$$
\begin{aligned}
\mathbf{y}_{2} & =\beta\left(\sqrt{2 P_{1}} \mathbf{S}_{1} \mathbf{h}+\sqrt{2 P_{2}} \mathbf{S}_{2} \mathbf{g}\right)+\mathbf{w}_{2} \\
& =\beta\left(\sqrt{2 P_{1}} \mathbf{H s}_{1}+\sqrt{2 P_{2}} \mathbf{G}_{2}\right)+\mathbf{w}_{2}
\end{aligned}
$$

where $\mathbf{H}, \mathbf{S}_{2}$, and $\mathbf{w}_{2}$ are defined similarly as in (7), and

$$
\mathbf{g}=\left[g_{1}^{2}, \ldots, g_{N}^{2}\right]^{T}, \quad \mathbf{G}=\sum_{i=1}^{N} g_{i}^{2} \mathbf{A}_{r, i} \mathbf{A}_{2}
$$

Since the true $\mathbf{S}_{m}$ is known at $\mathbb{T}_{m}$, the ML decoding of (17) can be easily obtained as

$$
\hat{\mathbf{s}}_{1}=\underset{\tilde{\mathbf{s}}_{1} \in \mathcal{A}_{1}^{N}}{\operatorname{argmin}}\left\|\mathbf{y}_{2}-\beta\left(\sqrt{2 P_{1}} \mathbf{H} \tilde{\mathbf{s}}_{1}+\sqrt{2 P_{2}} \mathbf{G s}_{2}\right)\right\|^{2}
$$

which can be solved by using sphere decoder [15]. Like the 4-AF protocol, we can simply set $\mathbf{A}_{1}=\mathbf{I}_{K}$. The operation at terminal $\mathbb{T}_{1}$ can be obtained similarly [see (20) shown at the bottom of the page].

\section{B. Partial Decode-and-Forward I (PDF I)}

Noting that the 2 -AF protocol amplifies the relay noise, we propose a new protocol to mitigate this effect. Instead of simply amplifying the received signal, $\mathbb{R}_{i}$ first decodes $\mathbf{s}_{1}$ and $\mathbf{s}_{2}$ via the ML decoder

$$
\begin{aligned}
& \left\{\hat{\mathbf{s}}_{1, i}, \hat{\mathbf{s}}_{2, i}\right\} \\
& \quad=\underset{\tilde{\mathbf{s}}_{1} \in \mathcal{A}_{1}^{N}, \tilde{\mathbf{s}}_{2} \in \mathcal{A}_{2}^{N}}{\operatorname{argmin}}\left\|\mathbf{y}_{r, i}-\sqrt{2 P_{1}} f_{i} \mathbf{A}_{1} \tilde{\mathbf{s}}_{1}-\sqrt{2 P_{2}} g_{i} \mathbf{A}_{2} \tilde{\mathbf{s}}_{2}\right\|_{2}^{2} .
\end{aligned}
$$

Note that (21) represents an under-determined system, where the number of unknowns is twice of the number of equations. Even though (21) could be efficiently solved using generalized sphere decoder [16], the high decoding complexity could be a practical issue. Moreover, the error probability of the under-determined system (21), even with ML decoding, is still high. Therefore, it is not good to send $\hat{\mathbf{s}}_{1, i}$ and $\hat{\mathbf{s}}_{2, i}$ directly. To mitigate error propagation, we propose that each relay sends

$$
\mathbf{x}_{r, i}=\mathbf{A}_{r, i}\left(\sqrt{2 P_{1}} f_{i} \hat{\mathbf{s}}_{1, i}+\sqrt{2 P_{2}} g_{i} \hat{\mathbf{s}}_{2, i}\right)
$$

after being scaled by $\beta=\beta_{i}=\sqrt{P_{3} / N\left(P_{1}+P_{2}\right)}$. Note that we still use the form $\sqrt{2 P_{1}} f_{i} \hat{\mathbf{s}}_{1, i}+\sqrt{2 P_{2}} g_{i} \hat{\mathbf{s}}_{2, i}$ as the useful signal component in the received signal. This scheme can be considered as removing noise from the received signal while keeping the channel effect, and is thus named as partial decodeand-forward (PDF). Note that the relay could also decode $\mathbf{x}_{r, i}$ directly rather than decode $\mathbf{s}_{1}$ and $\mathbf{s}_{2}$ separately. For example, when $f_{i}=g_{i}=1$ and both terminals use BPSK, the relay only sees a ternary constellation $\{-2,0,2\}$. Although decoding $\mathbf{x}_{r, i}$ directly reduces complexity, in fading channels the probability that such constellation compression happens with probability 0 . Therefore, with probability 1 , decoding $\mathbf{x}_{r, i}$ has the same complexity as decoding $\mathbf{s}_{1}$ and $\mathbf{s}_{2}$.

Let $P\left(\Delta \mathbf{s}_{1, i}, \Delta \mathbf{s}_{2, i}\right)$ denote the pairwise error probability at $\mathbb{R}_{i}$, where $\Delta \mathbf{s}_{1, i}=\mathbf{s}_{1}-\hat{\mathbf{s}}_{1, i}$ and $\Delta \mathbf{s}_{2, i}=\mathbf{s}_{2}-\hat{\mathbf{s}}_{2, i}$. The ML decoder at $\mathbb{T}_{2}$ can be obtained as (20) at the top of this page, where $\mathbf{H}$ and $\mathbf{G}$ are defined in (17). When $N$ or the constellation size is large, it is hard to implement (20) directly. In high signal-to-noise ratio (SNR), $\prod_{i=1}^{N} P\left(\Delta \mathbf{s}_{1, i}, \Delta \mathbf{s}_{2, i}\right)$ is dominated by $\Delta \mathbf{s}_{1, i}=\mathbf{0}, \Delta \mathbf{s}_{2, i}=\mathbf{0}, \forall i$. Thus, we approximate the ML decoding at $\mathbb{T}_{2}$ by

$$
\hat{\mathbf{s}}_{1}=\underset{\tilde{\mathbf{s}}_{1} \in \mathcal{A}_{1}^{N}}{\operatorname{argmin}}\left\|\mathbf{y}_{2}-\beta\left(\sqrt{2 P_{1}} \mathbf{H} \tilde{\mathbf{s}}_{1}+\sqrt{2 P_{2}} \mathbf{G s}_{2}\right)\right\|^{2} \text {. }
$$

$$
\begin{aligned}
\hat{\mathbf{s}}_{1}=\arg \max _{\mathbf{s}_{1} \in \mathcal{A}_{1}^{N}} \sum_{\Delta \mathbf{s}_{1, i}, \Delta \mathbf{s}_{2, i}} \exp \{ & -\| \mathbf{y}_{2}+\sum_{i=1}^{N} \beta \mathbf{A}_{r, i}\left(\sqrt{2 P_{1}} f_{i} \mathbf{A}_{1} \Delta \mathbf{s}_{1, i}+\sqrt{2 P_{2}} g_{i} \mathbf{A}_{2} \Delta \mathbf{s}_{2, i}\right) \\
& \left.-\beta\left(\sqrt{2 P_{1}} \mathbf{H} \tilde{\mathbf{s}}_{1}+\sqrt{2 P_{2}} \mathbf{G s}_{2}\right) \|_{2}^{2}\right\} \prod_{i=1}^{N} P\left(\Delta \mathbf{s}_{1, i}, \Delta \mathbf{s}_{2, i}\right),
\end{aligned}
$$


Note that by the ML decoder at $\mathbb{R}_{i}(21)$, we have

$$
\begin{array}{r}
\left\|\sqrt{2 P_{1}} f_{i} \mathbf{A}_{1}\left(\mathbf{s}_{1}-\hat{\mathbf{s}}_{1}\right)+\sqrt{2 P_{2}} g_{i} \mathbf{A}_{2}\left(\mathbf{s}_{2}-\hat{\mathbf{s}}_{2}\right)+\mathbf{n}_{r, i}\right\|_{2} \\
\leq\left\|\mathbf{n}_{r, i}\right\|_{2} .
\end{array}
$$

Therefore, the error between the correct signal without noise and the signal transmitted by PDF I is bounded.

The decoder (23) does not require knowledge of the PEP at the terminal and it only contains a single term. Thus, the decoding complexity is greatly decreased. Even though DF protocol performs worse than AF protocol in one way relay networks [17], we found in simulations that the two way relay PDF I protocol performs better than AF protocol even with the suboptimal decoder (23). The only limitation of PDF I protocol is that it may not satisfy peak power constraint in some cases because $\mathbf{x}_{r, i}$ in (22) depends on $f_{i}$ and $g_{i}$.

\section{Partial Decode-and-Forward II (PDF II)}

Both AF and PDF I transmit a weighted sum of signals from two terminals. However, this is wasteful in terms of the power consumption as $\mathbb{T}_{m}$ already knows $\mathbf{s}_{m}, m=1,2$. In PDF II, we propose to superimpose the signals via modular arithmetic. Let $M_{m}$ denote the size of the constellation $\mathcal{A}_{m}$, and $\mathcal{A}_{m}(j)$ denote the $j$ th element of $\mathcal{A}_{m}, m=1,2, j=0, \ldots, M_{m}-1$. Define $\mathbf{v}_{1}$ and $\mathbf{v}_{2}$ such that $\mathcal{A}_{1}\left(\mathbf{v}_{1}\right)=\mathbf{s}_{1}$ and $\mathcal{A}_{2}\left(\mathbf{v}_{2}\right)=\mathbf{s}_{2}$. Without loss of generality, we assume that $M=M_{1} \geq M_{2}$.

In this protocol, each relay obtains $\hat{\mathbf{s}}_{1, i}, \hat{\mathbf{s}}_{2, i}$ from (21) as in PDF I. Let $\mathcal{A}_{1}\left(\hat{\mathbf{v}}_{1, i}\right)=\hat{\mathbf{s}}_{1, i}$ and $\mathcal{A}_{2}\left(\hat{\mathbf{v}}_{2, i}\right)=\hat{\mathbf{s}}_{2, i}$. In this case

$$
\mathbf{x}_{r, i}=\mathbf{A}_{r, i} \mathcal{A}_{1}\left(\bmod \left(\hat{\mathbf{v}}_{1, i}+\hat{\mathbf{v}}_{2, i}, M\right)\right)
$$

where mod denotes the componentwise modular operation ${ }^{2}$ and $\beta=\beta_{i}=\sqrt{\left(2 P_{3} / N\right)}$. Since fading channels are considered, the probability that there exists a pair of vectors $\left\{\mathbf{v}_{1}, \mathbf{v}_{2}\right\}$ and $\left\{\hat{\mathbf{v}}_{1}, \hat{\mathbf{v}}_{2}\right\}$ such that $\sqrt{2 P_{1}} f_{i} \mathcal{A}_{1}\left(\mathbf{v}_{1}\right)+\sqrt{2 P_{2}} g_{i} \mathcal{A}_{2}\left(\mathbf{v}_{2}\right)=$ $\sqrt{2 P_{1}} f_{i} \mathcal{A}_{1}\left(\hat{\mathbf{v}}_{1}\right)+\sqrt{2 P_{2}} g_{i} \mathcal{A}_{2}\left(\hat{\mathbf{v}}_{2}\right)$ is vanishingly small. The relay uses $\mathcal{A}_{1}$ to ensure that when each terminal decodes $\mathbf{x}_{r, i}$ correctly there does not exist $\mathbf{v}_{1}, \mathbf{v}_{1}^{\prime}, \mathbf{v}_{1} \neq \mathbf{v}_{1}^{\prime}$ such that $\bmod \left(\mathbf{v}_{1}+\mathbf{v}_{2}, M\right)=\bmod \left(\mathbf{v}_{1}^{\prime}+\mathbf{v}_{2}, M\right)$, which removes the decoding ambiguity. Actually, the relay could use any other constellation of size greater than $M_{1}$.

As in (20), the true ML decoder at $\mathbb{T}_{2}$ can be obtained by considering all the PEPs at the relay. For simplicity, we approximate the ML decoding at $\mathbb{T}_{2}$ by

$\hat{\mathbf{s}}_{1}=\underset{\tilde{\mathbf{s}}_{1}=\mathcal{A}_{1}^{N}\left(\tilde{\mathbf{v}}_{1}\right)}{\operatorname{argmin}}\left\|\mathbf{y}_{2}-\beta \sum_{i=1}^{N} g_{i} \mathbf{A}_{r, i} \mathcal{A}_{1}\left(\bmod \left(\tilde{\mathbf{v}}_{1}+\mathbf{v}_{2}, M\right)\right)\right\|^{2}$.

We could first decode $\hat{\mathbf{x}}_{2}$ from $\hat{\mathbf{x}}_{2}=\bmod \left(\tilde{\mathbf{v}}_{1}+\mathbf{v}_{2}, M\right)$ via sphere decoder, and then decode $\hat{\mathbf{s}}_{1}$ from $\hat{\mathbf{s}}_{1}=$ $\bmod \left(\hat{\mathbf{x}}_{2}-\mathbf{v}_{2}, M\right)$.

Compared with (22), power in PDF II is saved from the modular operation. This scheme looks similar to network coding but has two main differences: 1) the scheme is applied at the phys-

\footnotetext{
${ }^{2}$ In this paper, $\bmod (a, n)$ means the remainder of $a$ divided by $n$ that lies in
} $[0, n)$. ical layer but network coding is performed at the network layer; 2) the operation in (25) is on a modular group, whereas finite field is used in network coding.

To exploit the diversity offered by multiple relays, we assume that each relay is able to determine whether $\bmod \left(\hat{\mathbf{v}}_{1, i}+\hat{\mathbf{v}}_{2, i}, M\right)=\bmod \left(\mathbf{v}_{1}+\mathbf{v}_{2}, M\right)$ through the use of CRCs or other error detecting codes. When $M=2$, the XOR between CRCs of $\mathbf{v}_{1}$ and $\mathbf{v}_{2}$ is also the CRC of $\bmod \left(\mathbf{v}_{1}+\mathbf{v}_{2}, M\right)$. Note that this is different from checking the correctness of $\mathbf{v}_{1}$ or $\mathbf{v}_{2}$ individually as both of them may be wrong though their modular sum is correct. Each relay will send $\mathbf{x}_{r, i}$ in (25) if and only if the modular sum is correct. With the correct modular sum and the known $\mathbf{v}_{i}$, each terminal can decode its desired signal. This can potentially improve the system performance. The terminal decoder can be obtained similarly as (20) and (23). In the following, we only consider the suboptimal decoder with the same form as (23).

Let us look at a simple example with a single relay as [18] to compare the two PDF schemes. Let $K=1, f=g=1$, and $P_{1}=P_{2}=1 / 2$. We assume BPSK at both terminals with $\mathcal{A}_{m}(0)=-1, \mathcal{A}_{m}(1)=1, m=1,2$. From (15), the relay receives $y_{r}=s_{1}+s_{2}+n_{r}$. Note that the decoder (21) cannot distinguish between $s_{1}=1, s_{2}=-1$ and $s_{1}=-1, s_{2}=1$. If $n_{r}$ is small, in either case, PDF I will transmit 0 and PDF II will transmit 1 as $\mathcal{A}_{1}(\bmod (0+1,2))=1$. Both protocols ensure correct decoding at two end terminals even if the transmitted signal by the relay contains ambiguity. The main difference between the two protocols appears when $s_{1}=1, s_{2}=1$ or $s_{1}=-1, s_{2}=-1$. With fixed transmit power 1 at the relay, PDF I will transmit $\sqrt{2}$ and $-\sqrt{2}$ in the two cases, while PDF II will transmit -1 in both cases. Given $s_{2}$, the Euclidean distance between the correct $s_{1}$ and its nearest neighbor in PDF I is $\sqrt{2}$, while this distance increases to 2 in PDF II. Therefore, PDF II has 3-dB expected gain over PDF I in this example. The gain actually comes from the modular operation. There may be some specially designed constellations for PDF II that could further improve the performance. Addressing constellation design is beyond the scope of this paper. We simply use the existing constellations, e.g., BPSK, QPSK, etc., in this paper.

\section{Practical Issues}

In practical networks, data is bursty, and there may not consistently be traffic in both directions. The relay nodes can detect two-way traffic by monitoring the average power of the received signal. If a relay determines that there is no two-way traffic, it simply uses the protocols for one-way traffic; otherwise, it switches to two-way mode discussed in this section. Also, we do not need perfect packet-level synchronization. By the energy detector, each relay can determine which received symbols suffer from interference due to two-way traffic, and it performs the corresponding operations.

In the PDF II protocol, each terminal also needs to know whether a particular relay transmits in a time slot. This can be realized by transmitting a beacon signal from the relay to the terminals at the beginning of each time slot to indicate that the relay will transmit data in this time slot. In OFDM based networks, this beacon signal can be transmitted through a certain subcarrier. 
When each relay uses a random unitary matrix, we can absorb this matrix into the channel, and the terminals can estimate the equivalent channel directly by using the algorithms in [13]. Another way is that each relay transmits to both terminals the random seed used to generate the random matrices.

Remarks:

- A comparison of this protocol with random linear network coding [11] is in place. By using network coding, each relay transmits a random linear combination of the received signals, where the operations are on a finite field. As network coding is applied on the network layer, received signals are assumed to be error free. By applying precoding matrices $\mathbf{A}_{1}$ and $\mathbf{A}_{2}$, addition is automatically done at the relay. The denoising process can also remove part of the noise. If we further choose $\mathbf{A}_{1}$ and $\mathbf{A}_{2}$ to be random unitary matrices, the proposed PDF protocol is similar to random linear network coding and can be considered as analog network coding operating at physical layer. Here the unitary matrix group plays the same role as the finite field in random network coding.

- Note that PDF I is not limited to two-way networks. It can also be applied to general networks such as multiple-layer relay networks, where instead of decoding the received signal at each node, we only apply the denoising process. Also, it can be easily seen that PDF I reduces to the modified DF when only one terminal transmits.

- The proposed protocols can be readily extended to the case where each node is equipped with multiple antennas as in [19].

- When the channel is unbalanced, e.g., one of $f_{i}, g_{i}$ is much greater than the other or the variance of one is much greater than the other, the error probabilities of $\mathbf{s}_{1}$ and $\mathbf{s}_{2}$ are not equal. The probability of correct decoding after modular operation in PDF II is limited by the weaker channel. But PDF I does not have this problem as seen from simulations because in PDF I the estimation of the two terminal signals are weighted by the channel coefficients.

- PDF II has a better performance over PDF I in simulations, but PDF I does not require CRC as PDF II does.

- Another feature of the proposed two-time-slot protocols is that they require minimal synchronization and have a small coordination overhead. In addition to having higher spectral efficiency, the proposed protocols also have MAC layer gains. We will elaborate on this point more in Section VI.

- By using random unitary matrix at each relay, the proposed protocols are fully decentralized, i.e., no coordination is needed between the terminals and the relays to determine the space time codes used at each relay. They also do not need to know the number of participating relays. In other words, each relay's operations do not depend on the network parameters. However, the terminals need to know all $\mathbf{A}_{r, i}$ for decoding. Also in PDF II, the terminals need to know the number of cooperating relays.

- PDF I uses the unitary matrix multiplicative group, while PDF II adopts the modular additive group. The finite field in network coding contains both a multiplicative group and an additive group. It is interesting to see if these two protocols can be combined.
- Except using random unitary matrices and optimizing the precoding matrices by minimizing the PEP as in Section VI, the precoding matrices can also be designed by maximizing the mutual information for AF based protocols directly as in [12].

\section{DSTC Protocols Using Three Time Slots}

In this section, we consider three-time-slot protocols. In the first time slot, $\mathbb{T}_{1}$ transmits and in the second time slot, $\mathbb{T}_{2}$ transmits. The transmission power is $\sqrt{3 P_{i}}$ because each terminal transmits every three time slots. The received signal at $\mathbb{R}_{i}$ in the first and the second time slots are

$$
\begin{aligned}
& \mathbf{y}_{i}^{r, 1}=\sqrt{3 P_{1}} f_{i} \mathbf{s}_{1}+\mathbf{n}_{r, i}^{(1)} \\
& \mathbf{y}_{i}^{r, 2}=\sqrt{3 P_{2}} g_{i} \mathbf{s}_{2}+\mathbf{n}_{r, i}^{(2)}
\end{aligned}
$$

respectively, where $\mathbf{y}_{i}^{r, 1}, \mathbf{y}_{i}^{r, 2}, \mathbf{n}_{r, i}^{(1)}$, and $\mathbf{n}_{r, i}^{(2)}$ are defined similar to (5). In the third time slot, each $\mathbb{R}_{i}$ transmits $\mathbf{x}_{r, i}$ scaled by $\beta_{i}$ to meet its power constraint. The received signals at $\mathbb{T}_{1}$ and $\mathbb{T}_{2}$ are the same as those in (16).

The three-time-slot protocols require coordination between the two terminals to determine which one should transmit at a given time slot, but the three-time-slot protocols have an advantage over the two-time-slot protocols in that they can exploit the direct transmission between the two terminals.

\section{A. Amplify-and-Forward (3-AF)}

At each relay, a linear combination of $\mathbf{y}_{i}^{r, 1}$ and $\mathbf{y}_{i}^{r, 2}$ is first precoded by a unitary matrix $\mathbf{A}_{r, i}$ and is then scaled by a factor $\beta_{i}$ to satisfy the average power constraint, which gives the received signal at the terminal $\mathbb{T}_{2}$ as

$$
\mathbf{y}_{2}=\left(\sqrt{3 P_{1}} \mathbf{S}_{1} \mathbf{B} \mathbf{\Lambda h}+\sqrt{3 P_{2}} \mathbf{S}_{2} \mathbf{B}(\mathbf{I}-\mathbf{\Lambda}) \mathbf{g}\right)+\mathbf{w}_{2}
$$

where $\boldsymbol{\Lambda}=\operatorname{diag}\left\{\sqrt{\lambda_{1}}, \ldots, \sqrt{\lambda_{N}}\right\}$ with $0 \leq \lambda_{i} \leq 1$ being a power allocation coefficient at $\mathbb{R}_{i}, \mathbf{B}=\operatorname{diag}\left\{\beta_{1}, \ldots, \beta_{N}\right\}$ with $\beta_{i}=\sqrt{\left(3 P_{3} / N\left(3 \lambda_{i} P_{1}+3\left(1-\lambda_{i}\right) P_{2}+1\right)\right)}$, and

$$
\begin{aligned}
\mathbf{w}_{2}=\left(\sum_{i=1}^{N} \beta_{i} \sqrt{\lambda_{i}} g_{i} \mathbf{A}_{r, i} \mathbf{n}_{r, i}^{(2)}\right. & \\
& \left.+\sum_{i=1}^{N} \beta_{i} \sqrt{1-\lambda_{i}} g_{i} \mathbf{A}_{r, i} \mathbf{n}_{r, i}^{(1)}\right)+\mathbf{n}_{2} .
\end{aligned}
$$

In addition, $\mathbf{S}_{i}, \mathbf{g}$ are defined similarly as in (17). The ML decoder at the two terminals can be obtained as (19). Compared with 4-AF and 2-AF, the 3-AF protocol in this section suffers from more noise amplification as it actually adds the noise in the first and the second time slots together. Therefore, this protocol has poor performance and is not preferred in practice.

\section{B. Decode-and-Forward I (3-DF I)}

As the DF protocol in Section III, each $\mathbb{R}_{i}$ decodes $\mathbf{s}_{1}, \mathbf{s}_{2}$ by using (13) after the first and the second time slots. A linear combination of $\hat{\mathbf{s}}_{1, i}$ and $\hat{\mathbf{s}}_{2, i}$ is then precoded by a unitary matrix $\mathbf{A}_{r, i}$ and is scaled by a factor $\beta_{i}$ to satisfy the average power constraint, which gives the transmitted signal at $\mathbb{R}_{i}$ as

$$
\mathbf{x}_{r, i}=\sqrt{\frac{3 P_{3}}{N}} \mathbf{A}_{r, i}\left(\sqrt{\lambda_{i}} f_{i} \hat{\mathbf{s}}_{1, i}+\sqrt{1-\lambda_{i}} g_{i} \hat{\mathbf{s}}_{2, i}\right) .
$$


One advantage of 3-DF I over the two time slots protocols is that each relay can flexibly change the power allocation to the signals from two terminals.

\section{Decode-and-Forward II (3-DF II)}

This protocol extends PDF II in Section IV. After decoding $\mathbf{s}_{1}, \mathbf{s}_{2}$ by using (13), we find the index vectors $\hat{\mathbf{v}}_{1, i}, \hat{\mathbf{v}}_{2, i}$ such that $\mathcal{A}_{1}\left(\hat{\mathbf{v}}_{1, i}\right)=\hat{\mathbf{s}}_{1, i}$ and $\mathcal{A}_{2}\left(\hat{\mathbf{v}}_{2, i}\right)=\hat{\mathbf{s}}_{2, i}$. As in PDF II, each relay $i$ then sends

$$
\mathbf{x}_{r, i}=\sqrt{\frac{3 P_{3}}{N}} \mathbf{A}_{r, i} \mathcal{A}_{1}\left(\bmod \left(\hat{\mathbf{v}}_{1, i}+\hat{\mathbf{v}}_{2, i}, M\right)\right) .
$$

Let $d$ denote the channel gain between the two terminals. Moreover, let $\mathbf{y}_{2}^{1}$ and $\mathbf{y}_{2}^{3}$ denote the received signal at $\mathbb{T}_{2}$ in time slot 1 and 3 respectively. In case of the 3-DF I protocol, the approximate ML decoder at $\mathbb{T}_{2}$ can be obtained as

$$
\begin{array}{r}
\hat{\mathbf{s}}_{1}=\underset{\tilde{\mathbf{s}}_{1} \in \mathcal{A}_{1}^{N}}{\operatorname{argmin}}\left\|\mathbf{y}_{2}^{3}-\sqrt{\frac{3 P_{3}}{N}}\left(\boldsymbol{\Theta}_{1} \tilde{\mathbf{s}}_{1}+\boldsymbol{\Theta}_{2} \mathbf{s}_{2}\right)\right\|_{2}^{2} \\
+\left\|\mathbf{y}_{2}^{1}-d \sqrt{3 P_{1}} \mathbf{A}_{1} \tilde{\mathbf{s}}_{1}\right\|_{2}^{2}
\end{array}
$$

where

$$
\boldsymbol{\Theta}_{1}=\sum_{i=1}^{N} \lambda_{i} f_{i} g_{i} \mathbf{A}_{r, i}, \quad \boldsymbol{\Theta}_{2}=\sum_{i=1}^{N}\left(1-\lambda_{i}\right) g_{i}^{2} \mathbf{A}_{r, i} .
$$

The ML decoder at $\mathbb{T}_{1}$ and for 3-DF II can be obtained similarly. Therefore, the three-time-slot protocols can exploit the benefit offered by the direct transmission between the two terminals, which may be useful when the direct link is strong and the number of relays is small.

\section{PERFORMANCE ANALYSIS AND OPTIMIZATION}

In this section, we analyze the pairwise error probability (PEP) of the proposed two-time-slot protocols, and compare them with four-time-slot protocols. Our focus in this section is deriving the achievable diversity order and coding gain for different protocols. For brevity, we only analyze some representative protocols.

\section{A. Amplify-and-Forward}

We consider the PEP of mistaking $\mathbf{s}_{m}$ by $\mathbf{s}_{m}^{\prime}$ and define $\boldsymbol{\Delta}_{m}=\mathbf{s}_{m}-\mathbf{s}_{m}^{\prime}, \mathbf{C}_{m}=\left[\mathbf{A}_{r, 1} \boldsymbol{\Delta}_{m}, \ldots, \mathbf{A}_{r, N} \boldsymbol{\Delta}_{m}\right]$, $\mathbf{M}_{m}=\mathbf{C}_{m}^{H} \mathbf{C}_{m} m=1,2$. Let $\mu_{m}$ denote the rank of $\mathbf{M}_{m}$ and $P=P_{1}+P_{2}+P_{3}$ denote the total average power of the whole network. As an example, we have the following theorem on the performance of the 2-AF protocol in Section IV.

Theorem 1: For the 2-AF protocol, if $\log P \gg 1, K \geq N$, and $N \gg 1$, the sum of PEPs of the two terminals is minimized when $P_{1}=P_{2}=P / 4$ and $P_{3}=P / 2$. With this power allocation, the PEPs of signals from $\mathbb{T}_{m}, m=1,2$ are upper bounded by

$$
\mathrm{PEP}_{m} \lesssim \operatorname{det}^{-1} \mathbf{M}_{m}\left(\frac{8 N}{K}\right)^{\mu_{m}} P^{-\mu_{m}(1-\log \log P / \log P)}
$$

where $\lesssim$ means $\leq$ almost surely [2]
Proof: After canceling the contribution of its transmitted signal, each terminal sees a one-way relay channel. By using [2, Theorem 1] for AF in one-way relay channel and considering the expression of (17), we obtain

$$
\begin{gathered}
\mathrm{PEP}_{1}+\mathrm{PEP}_{2} \leq \underset{g_{i}}{\mathrm{E}_{i}} \operatorname{det}^{-1}\left[\mathbf{I}_{N}+\frac{P_{1} P_{3}}{N(1+2 P)} \mathbf{M}_{1} \operatorname{diag}(\mathbf{g})\right] \\
+\underset{f_{i}}{\mathrm{E} \operatorname{det}^{-1}}\left[\mathbf{I}_{N}+\frac{P_{2} P_{3}}{N(1+2 P)} \mathbf{M}_{2} \operatorname{diag}(\mathbf{f})\right]
\end{gathered}
$$

where $\mathbf{f}=\left[\left|f_{1}\right|^{2}, \ldots,\left|f_{N}\right|^{2}\right]^{T}$. First note that (36) is a convex function in $P_{1}$ and $P_{2}$, given $P_{3}$. Since $P$ is fixed, $P_{1}+P_{2}$ is also fixed, given $P_{3}$. Therefore, (36) is minimized when $P_{1}=P_{2}$ by assuming $\mathbf{M}_{1}=\mathbf{M}_{2}$. By minimizing (36) under the conditions $P_{1}=P_{2}$ and $P=P_{1}+P_{2}+P_{3}$, we get the optimal power allocation as $P_{1}=P_{2}=P / 4$ and $P_{3}=P / 2$. By using [2, Corollary 2], which approximates $\mathrm{PEP}_{m}$ by

$$
\mathrm{PEP}_{m} \lesssim \operatorname{det}^{-1} \mathbf{M}_{m}\left(\frac{8 N}{K}\right)^{\mu_{m}} P^{-\mu_{m}(1-(\log \log P / \log P))}
$$

$m=1,2$, we obtain (35).

From (35), it is clear that the optimal codes $\mathbf{A}_{i}, i=1, \ldots, N$ should maximize the minimum $\operatorname{det} \mathbf{M}_{m}, m=1,2$. The following theorem provides a sufficient condition on the optimal design.

Theorem 2: For the 2-AF protocol, if $K \geq N$, a set of matrices $\mathbf{A}_{r, i}, i=1, \ldots, N$ achieves the minimum PEP if $\operatorname{det} \mathbf{M}_{m}=\left\|\Delta_{m}\right\|_{2}^{2 N}$ and diversity order $N(1-(\log \log P / \log P))$ can be achieved.

Proof: Due to the symmetry, we drop the subscript on $\mathbf{M}_{m}$ and $\boldsymbol{\Delta}_{m}$. Given any $\boldsymbol{\Delta}$, by Householder transformation [20], there exists a unitary matrix $\mathbf{U}$ such that $\mathbf{U} \boldsymbol{\Delta}=\left[\|\Delta\|_{2}, 0, \ldots, 0\right]^{T}$. Let $\tilde{\mathbf{A}}_{i}=\mathbf{A}_{r, i} \mathbf{U}^{H}$ be a new unitary matrix, and $\tilde{\mathbf{a}}_{i}$ be its first column with $\left\|\tilde{\mathbf{a}}_{i}\right\|_{2}=1$. Denote the matrix $\mathbf{D}=\left[\tilde{\mathbf{a}}_{1}, \ldots, \tilde{\mathbf{a}}_{N}\right]$. It is easy to show that $\mathbf{M}=\|\Delta\|_{2}^{2} \mathbf{D}^{H} \mathbf{D}$. Note that the diagonal entries of $\mathbf{D}^{H} \mathbf{D}$ are all ones and $\mathbf{D}^{H} \mathbf{D}$ is positive semidefinite. By Hadamard inequality, we obtain $\operatorname{det} \mathbf{M} \leq\|\Delta\|_{2}^{2 N}$. If $\operatorname{det} \mathbf{M}=\|\Delta\|_{2}^{2 N}$, then the rank of $\mathbf{M}$ is $N$. By Theorem 1 , the diversity order is $N(1-(\log \log P / \log P))$.

From Theorem 2, it is clear that if $\mathbf{S}=\left[\mathbf{A}_{r, 1} \mathbf{s}, \ldots, \mathbf{A}_{r, N} \mathbf{S}\right]$ constitutes an orthogonal space time code, then it achieves the minimum PEP. For example, when $N=K=2$ and $\mathcal{A}$ is a real set, we can choose

$$
\mathbf{A}_{r, 1}=\left[\begin{array}{ll}
1 & 0 \\
0 & 1
\end{array}\right], \quad \mathbf{A}_{r, 2}=\left[\begin{array}{cc}
0 & 1 \\
-1 & 0
\end{array}\right] .
$$

It is easy to show that Theorem 2 is satisfied with (38). In fact, (38) is a variant of Alamouti code.

When $\mathbf{A}_{r, i}, i=1, \ldots, N$ are all random unitary matrices, it is known [2] that the diversity order $N(1-(\log \log P / \log P))$ can be achieved if the following two conditions hold:

- the matrix $\mathbf{M}_{m}$ is full rank with high probability;

- the expectation $\mathrm{E}\left\{\operatorname{det}^{-1} \mathbf{M}\right\}$ is finite.

By using the same approach as the proof of Theorem 2 and noting that $\tilde{\mathbf{A}}_{i}=\mathbf{A}_{r, i} \mathbf{U}^{H}$ is also a random unitary matrix, we can show that verifying the above two conditions is equivalent to showing whether $\mathbf{D}^{H} \mathbf{D}$ is full rank and whether 
$\mathrm{E}\left\{\operatorname{det}^{-1} \mathbf{D}^{H} \mathbf{D}\right\}$ is finite, where each column is drawn uniformly and independently on the complex hypersphere with unit radius. When $N=K=2$, it can be shown that the eigenvalues of $\mathbf{D}^{H} \mathbf{D}$ are $1-\sqrt{\xi}$ and $1+\sqrt{\xi}$, where $\xi \sim F_{24}$ and $F_{n m}$ is the $F$-distribution. As $F_{24}$ is a continuous distribution, the eigenvalues are zeros with probability 0 . Therefore, the matrix $\mathbf{D}^{H} \mathbf{D}$ is full rank with probability 1 . Also, we can show that $1 / 1-\xi / 4$ is finite. Therefore, random unitary matrices achieve diversity order $2(1-(\log \log P / \log P))$.

The following theorem characterizes the SNR gain of 2-AF over 4-AF.

Theorem 3: Let $d_{\min }^{(4)}$ and $d_{\min }^{(2)}$ denote the minimum distance between points in the constellations used by $4-\mathrm{AF}$ and $2-\mathrm{AF}$, respectively, and $R^{(4)}$ and $R^{(2)}$ denote the rates of the two constellations. Denote $P^{(4)}$ and $P^{(2)}$ as the total power in the networks in the two cases. Assume that random unitary matrices or optimal unitary matrices are used with $K=N$. To achieve the same bit error rate (BER) in high SNR, we must have

$$
\frac{P^{(2)}}{P^{(4)}} \approx 2^{R^{(2)}-R^{(4)}+1^{N}} \sqrt{\frac{R^{(4)}}{R^{(2)}}}\left(\frac{d_{\min }^{(4)}}{d_{\min }^{(2)}}\right)^{2} .
$$

Proof: By following the proof of Theorem 1, we derive the PEP for 4-AF as

$\mathrm{PEP}_{i} \lesssim \operatorname{det}^{-1} \mathbf{M}_{i}\left(\frac{4 N}{K}\right)^{\mu_{i}} P^{-\mu_{i}(1-(\log \log P / \log P))}, \quad i=1,2$.

From Theorem 2, the worst case PEP of both (35) and (40) is attained when $\|\boldsymbol{\Delta}\|=d_{\min }$, where $d_{\min }$ is the minimum distance in a constellation. By using union bound and assuming that the diversity order $N$ can be attained and Gray mapping is used, we can obtain the bit error rate for $2-\mathrm{AF}$ as

$$
P_{b}^{(2)} \lesssim \frac{1}{R^{(2)}} 2^{N R^{(2)}}\left(d_{\mathrm{min}}^{(2)}\right)^{2 N}\left(\frac{8 N}{K}\right)^{N} P^{(2)-N} .
$$

The bit error rate $P_{b}^{(4)}$ for 4-AF can be obtained similarly by using (40) as

$$
P_{b}^{(4)} \lesssim \frac{1}{R^{(4)}} 2^{N R^{(4)}}\left(d_{\min }^{(4)}\right)^{2 N}\left(\frac{4 N}{K}\right)^{N} P^{(4)-N} .
$$

Comparing $P_{b}^{(2)}$ with $P_{b}^{(4)}$ proves the theorem.

Even though Theorem 3 is approximate due to the use of the union bound, this theorem can explain some interesting observations in our simulations. Though 4-AF requires more time slots than the two-time-slot counterpart, we can increase the constellation size in the 4-AF protocol to enhance the throughput. For example, we can choose 4-QAM in 4-AF and BPSK in 2-AF so that they attain the same throughput. In this case, $d_{\text {min }}^{(4)}=\sqrt{2}$, $d_{\min }^{(2)}=2, R^{(4)}=2$ and $R^{(2)}=1$. By Theorem 3, we ob$\operatorname{tain} P^{(2)} / P^{(4)} \approx \sqrt{[N] 2 / 2}$. Therefore, when $N$ is small, 2-AF does not have much power saving over 4-AF, which is also seen in the simulation results. This seems to contradict the intuition that the two-time-slot protocol saves power over the fourtime-slot protocol. We note that an average power constraint is assumed in this paper instead of peak power constraint as in, e.g., [2]. In 802.11 based protocols, the peak transmit power of each node is fixed. In this case, we can show that the two-timeslot protocol achieves a smaller BER than the four-time-slot protocol given the same throughput. However, the former consumes more power than the latter. As $d_{\mathrm{min}}^{(4)} / d_{\mathrm{min}}^{(2)}$ decreases by increasing the size of constellation, 2-AF requires less power than 4-AF when the required rate is high. Therefore, two-timeslot protocols are favorable in high rate communications.

\section{B. Partial Decode-and-Forward I}

In the following, we use the optimal power allocation between terminals and relay nodes obtained in Section VI-A, i.e., $P_{1}=$ $P_{2}=P / 4$ and $P_{3}=P / 2$, where $P$ is the total power of the network.

We consider the suboptimal decoder (23). Define $\Delta \mathbf{s}=\mathbf{s}_{1}-$ $\mathbf{s}_{1}^{\prime}, \Delta \mathbf{s}_{1, i}=\hat{\mathbf{s}}_{1, i}-\mathbf{s}_{1}$, and $\Delta \mathbf{s}_{2, i}=\hat{\mathbf{s}}_{2, i}-\mathbf{s}_{2}$. Due to the symmetry between the two terminals, we consider the pairwise error probability (PEP) of mistaking $\mathbf{s}_{1}$ by $\mathbf{s}_{1}^{\prime}$ conditioned on $f_{i}, g_{i}$, $\Delta \mathbf{s}_{1, i}, \Delta \mathbf{s}_{2, i}$, which is [see (43) on the next page], where $(a)$ comes from Chernoff bound, and

$$
\begin{aligned}
\mathbf{a}= & \sqrt{\frac{P}{2 N}} \sum_{i=1}^{N} f_{i} g_{i} \mathbf{A}_{r, i} \mathbf{A}_{1} \Delta \mathbf{s} \\
= & \sqrt{\frac{P}{2 N}} \mathbf{C} \tilde{\mathbf{G}} \tilde{\mathbf{f}} \\
\mathbf{b}= & \sqrt{\frac{P}{2 N}} \sum_{i=1}^{N} f_{i} g_{i} \mathbf{A}_{r, i} \mathbf{A}_{1} \Delta \mathbf{s}_{1, i} \\
& +\sqrt{\frac{P}{2 N}} \sum_{i=1}^{N} g_{i}^{2} \mathbf{A}_{r, i} \mathbf{A}_{1} \Delta \mathbf{s}_{2, i} \\
= & \sqrt{\frac{P}{2 N}} \sum_{i=1}^{N} g_{i} \mathbf{A}_{r, i} \mathbf{r}_{i}, \\
\mathbf{r}_{i}= & f_{i} \mathbf{A}_{1} \Delta \mathbf{s}_{1, i}+g_{i} \mathbf{A}_{2} \Delta \mathbf{s}_{2, i}, \\
\mathbf{C}= & {\left[\mathbf{A}_{r, 1} \mathbf{A}_{1} \Delta \mathbf{s}, \ldots, \mathbf{A}_{r, N} \mathbf{A}_{1} \Delta \mathbf{s}\right], } \\
\mathbf{C}_{j}= & {\left[\mathbf{A}_{r, 1} \mathbf{A}_{j} \Delta \mathbf{s}_{j, i}, \ldots, \mathbf{A}_{r, N} \mathbf{A}_{j} \Delta \mathbf{s}_{j, i}\right], } \\
j= & 1,2, \\
\tilde{\mathbf{f}}= & {\left[f_{1}, \ldots, f_{N}\right]^{T}, \quad \tilde{\mathbf{G}}=\operatorname{diag}\left\{g_{1}, \ldots, g_{N}\right\} . }
\end{aligned}
$$

On the other hand, the PEP at $\mathbb{R}_{i}$ can be easily obtained as

$$
P\left(\Delta \mathbf{s}_{1, i}, \Delta \mathbf{s}_{2, i}\right) \leq e^{-(P / 8)\left\|f_{i} \mathbf{A}_{1} \Delta \mathbf{s}_{1, i}+g_{i} \mathbf{A}_{2} \Delta \mathbf{s}_{2, i}\right\|^{2}} .
$$

The PEP conditioned on $f_{i}, g_{i}$ can thus be obtained as (45) shown on the next page, where we choose $\lambda=1 / 2\left(1+(4 / N) \sum_{i=1}^{N}\left|g_{i}\right|^{2}\right)$ in $(a)$. Integrating $P_{e}$ (pairwise $\mid f, g$ ) over $f, g$, we obtain (46), shown on the next page, where the last approximate inequality comes from [2, Corollary 2]. Therefore, the diversity order $N(1-(\log \log P / \log P))$ is achievable if $K \geq N$.

Different from the conventional DF scheme which cannot achieve diversity order greater than 1 [1], the proposed 2-PDF I protocol achieves the same diversity order as AF, which is observed from the simulation results in Section VII. This result also indicates that if we apply the PDF I protocol to one way relay networks, we can also obtain full diversity order. Note that 


$$
\begin{aligned}
& P_{e}\left(\text { pairwise } \mid f_{i}, g_{i}, \Delta \mathbf{s}_{1, i}, \Delta \mathbf{s}_{2, i}\right) \\
& =P\left\{\left\|\mathbf{y}_{2}-\sqrt{\frac{P_{3}}{N\left(P_{1}+P_{2}\right)}}\left(\sqrt{2 P_{1}} \mathbf{H} \hat{\mathbf{s}}_{1}+\sqrt{2 P_{2}} \mathbf{G s}_{2}\right)\right\|\right. \\
& \left.\leq\left\|\mathbf{y}_{2}-\sqrt{\frac{P_{3}}{N\left(P_{1}+P_{2}\right)}}\left(\sqrt{2 P_{1}} \mathbf{H s}_{1}+\sqrt{2 P_{2}} \mathbf{G s}_{2}\right)\right\|\right\} \\
& =P\left\{\| \sqrt{\frac{P}{2 N}} \sum_{i=1}^{N} f_{i} g_{i} \mathbf{A}_{r, i} \mathbf{A}_{1} \Delta \mathbf{s}+\sqrt{\frac{P}{2 N}} \sum_{i=1}^{N} f_{i} g_{i} \mathbf{A}_{r, i} \mathbf{A}_{1} \Delta \mathbf{s}_{1, i}\right. \\
& +\sqrt{\frac{P}{2 N}} \sum_{i=1}^{N} g_{i}^{2} \mathbf{A}_{r, i} \mathbf{A}_{1} \Delta \mathbf{s}_{2, i}+\mathbf{n}_{2} \|^{2} \\
& \left.\leq\left\|\sqrt{\frac{P}{2 N}} \sum_{i=1}^{N} f_{i} g_{i} \mathbf{A}_{r, i} \mathbf{A}_{1} \Delta \mathbf{s}_{1, i}+\sqrt{\frac{P}{2 N}} \sum_{i=1}^{N} g_{i}^{2} \mathbf{A}_{r, i} \mathbf{A}_{1} \Delta \mathbf{s}_{2, i}+\mathbf{n}_{2}\right\|^{2}\right\} \\
& =P\left\{\|\mathbf{a}\|^{2}+\mathbf{a}^{H} \mathbf{b}+\mathbf{b}^{H} \mathbf{a}+\mathbf{a}^{H} \mathbf{n}_{2}+\mathbf{n}_{2}^{H} \mathbf{a} \leq 0\right\} \\
& \stackrel{(a)}{\leq} E\left\{e^{-\lambda\left(\|\mathbf{a}\|^{2}+\mathbf{a}^{H} \mathbf{b}+\mathbf{b}^{H} \mathbf{a}+\mathbf{a}^{H} \mathbf{n}_{2}+\mathbf{n}_{2}^{H} \mathbf{a}\right)}\right\} \\
& =\int e^{-\lambda\left(\|\mathbf{a}\|^{2}+\mathbf{a}^{H} \mathbf{b}+\mathbf{b}^{H} \mathbf{a}+\mathbf{a}^{H} \mathbf{n}_{2}+\mathbf{n}_{2}^{H} \mathbf{a}\right)} e^{-\mathbf{n}_{2}^{H} \mathbf{n}_{2}} d \mathbf{n}_{2} \\
& =e^{-\lambda(1-\lambda)\|\mathbf{a}\|^{2}-\lambda\left(\mathbf{a}^{H} \mathbf{b}+\mathbf{b}^{H} \mathbf{a}\right)},
\end{aligned}
$$

$P_{e}\left(\right.$ pairwise $\left.\mid f_{i}, g_{i}\right)$

$$
\begin{aligned}
& =\sum_{\Delta \mathbf{s}_{1, i}, \Delta \mathbf{s}_{2, i}} P_{e}\left(\text { pairwise } \mid f_{i}, g_{i}, \Delta \mathbf{s}_{1, i}, \Delta \mathbf{s}_{2, i}\right) \prod_{i=1}^{N} P\left(\Delta \mathbf{s}_{1, i}, \Delta \mathbf{s}_{2, i}\right) \\
& \leq e^{-\lambda(1-\lambda)\|\mathbf{a}\|^{2}} \sum_{\Delta \mathbf{s}_{1, i}, \Delta \mathbf{s}_{2, i}} \exp \left(-\lambda\left(\sqrt{\frac{P}{2 N}} \sum_{i=1}^{N} g_{i} \mathbf{a}^{H} \mathbf{A}_{r, i} \mathbf{r}_{i}+g_{i}^{*} \mathbf{r}_{i}^{H}\left(\mathbf{A}_{r, i}\right)^{H} \mathbf{a}\right)-\frac{P}{8} \sum_{i=1}^{N}\left\|\mathbf{r}_{i}\right\|^{2}\right) \\
& =e^{-\lambda(1-\lambda)\|\mathbf{a}\|^{2}} \sum_{\Delta \mathbf{s}_{1, i}, \Delta \mathbf{s}_{2, i}} \exp \left(-\sum_{i=1}^{N}\left\|\sqrt{\frac{P}{8}} \mathbf{r}_{i}-\frac{2 \lambda}{\sqrt{N}}\left(\mathbf{A}_{r, i}\right)^{H} \mathbf{a}\right\|^{2}+\frac{4 \lambda^{2}}{N} \sum_{i=1}^{N}\left|g_{i}\right|^{2}\|\mathbf{a}\|^{2}\right) \\
& \left.\stackrel{(a)}{\leq} M_{1}^{K} M_{2}^{K} e^{-1 / 4(1+4 / N} \sum_{i=1}^{N}\left|g_{i}\right|^{2}\right)\|\mathbf{a}\|^{2}
\end{aligned}
$$

$$
\begin{aligned}
& E_{f_{i}, g_{i}}\left\{P_{e}\left(\text { pairwise } \mid f_{i}, g_{i}\right)\right\} \\
& \quad \leq E_{f_{i}, g_{i}}\left\{M_{1}^{K} M_{2}^{K} e^{-\left(1 / 4\left(1+4 / N \sum_{i=1}^{N}\left|g_{i}\right|^{2}\right)\right)\|\mathbf{a}\|^{2}}\right\} \\
& \quad=M_{1}^{K} M_{2}^{K} E_{g_{i}}\left\{-1\left[\mathbf{I}_{N}+\frac{1}{4\left(1+\frac{4}{N} \sum_{i=1}^{N}\left|g_{i}\right|^{2}\right)} \mathbf{C}^{H} \mathbf{C} \tilde{\mathbf{G}}^{H} \tilde{\mathbf{G}}\right]\right\} \\
& \quad \lesssim M_{1}^{K} M_{2}^{K} \operatorname{det}^{-1}\left(\mathbf{C}^{H} \mathbf{C}\right)\left(\frac{40 N}{P}\right)^{N} P^{-N(1-(\log \log P / \log P))}
\end{aligned}
$$


our performance analysis of 2-PDF I is imprecise because we use Chernoff bound. Detailed performance analysis may characterize the SNR gain of 2-PDF I over AF. Nevertheless, from the simulation results, we find that the achievable diversity order predicted by our analysis is correct.

\section{Partial Decode-and-Forward II}

For the PDF II protocol, we have the following theorem.

Theorem 4: When $K \geq N$, the PDF II protocol can attain the diversity order of $N$ with appropriate code design.

Proof: We first derive the PEP at each relay by taking $\left\{\mathbf{s}_{1}, \mathbf{s}_{2}\right\}$ to be $\left\{\mathbf{s}_{1}^{\prime}, \mathbf{s}_{2}^{\prime}\right\}$. Integrating (44) over $f_{i}$ and $g_{i}$, we obtain the upper bound of PEP at any relay as

$$
\mathrm{PEP} \leq \operatorname{det}^{-1}\left(\mathbf{I}_{2}+\frac{P}{16} \mathbf{M}\right)
$$

where $\mathbf{M}=\mathbf{C}^{H} \mathbf{C}, \mathbf{C}=\left[\mathbf{A}_{1} \Delta_{1}, \mathbf{A}_{2} \Delta_{2}\right]$, and $\Delta_{1}=\mathbf{s}_{1}-\mathbf{s}_{1}^{\prime}$, $\Delta_{2}=\mathbf{s}_{2}-\mathbf{s}_{2}^{\prime}$. By computing the right-hand side of (47), we obtain [see (48), shown at the bottom of the page].

From the inequality $\left\|\mathbf{x}_{1}\right\|_{2}^{2}\left\|\mathbf{x}_{2}\right\|_{2}^{2} \geq\left\|\mathbf{x}_{1}^{H} \mathbf{x}_{2}\right\|_{2}^{2}$, where equality is attained when $\mathbf{x}_{1}$ is a scaled version of $\mathbf{x}_{2}$, we get

$$
\operatorname{det}^{-1}\left(\mathbf{I}_{2}+\frac{P}{16} \mathbf{M}\right) \leq \frac{1}{1+\frac{P}{8}\|\mathbf{A} \Delta\|_{2}^{2}}
$$

where $\mathbf{A}_{1}=\mathbf{A}_{2}=\mathbf{A}$ and $\Delta_{1}=\Delta_{2}=\Delta$. To achieve a diversity order of 1 , we require that $\mathbf{A}_{i} \mathbf{s} \neq \mathbf{A}_{i} \mathbf{s}^{\prime}, \forall \mathbf{s} \neq \mathbf{s}^{\prime}, i=$ 1,2 . If $\mathbf{A}$ is unitary and $d_{\min }$ is the minimum distance of the constellation, we obtain

$$
\mathrm{PEP} \leq \frac{8}{P d_{\min }^{2}}
$$

Applying union bound on (50), we obtain the average error probability at $\mathbb{R}_{i}$ as

$$
P_{r, e} \leq M^{2 K} \frac{8}{P d_{\min }^{2}}
$$

where $\mathcal{A}_{1}=\mathcal{A}_{2}=M$.

Note that (51) is also a loose upperbound on the probability that $\bmod \left(\hat{\mathbf{v}}_{1, i}+\hat{\mathbf{v}}_{2, i}, M\right) \neq \bmod \left(\mathbf{v}_{1}+\mathbf{v}_{2}, M\right)$ at $\mathbb{R}_{i}$. If $k$ relays satisfy $\bmod \left(\hat{\mathbf{v}}_{1, i}+\hat{\mathbf{v}}_{2, i}, M\right)=\bmod \left(\mathbf{v}_{1}+\mathbf{v}_{2}, M\right)$, by following the approach in [21], we can bound the PEP of $\mathbf{s}_{1}$ as

$$
\mathrm{PEP}_{k} \leq \operatorname{det}^{-1}\left(\mathbf{I}_{k}+\frac{P_{3}}{2 N} \mathbf{M}\right)
$$

where $\mathbf{M}=\mathbf{C}^{H} \mathbf{C}, \mathbf{C}=\left[\mathbf{A}_{r, 1} \boldsymbol{\Delta}, \ldots, \mathbf{A}_{r, k} \boldsymbol{\Delta}\right]$, and $\boldsymbol{\Delta}=\mathcal{A}\left(\bmod \left(\mathbf{v}_{1}+\mathbf{v}_{2}, M\right)\right)-\mathcal{A}\left(\bmod \left(\hat{\mathbf{v}}_{1}+\mathbf{v}_{2}, M\right)\right)$. If orthogonal matrices are used, we obtain

$$
\mathrm{PEP}_{k} \leq\left(\frac{4 N}{P d_{\min }^{2}}\right)^{k}
$$

and the upperbound on average error probability is

$$
P_{e, k} \leq M^{K}\left(\frac{4 N}{P d_{\min }^{2}}\right)^{k}
$$

Finally, the overall error probability can be bounded as

$$
\begin{aligned}
P_{e} & \leq \sum_{k=0}^{N}\left(\begin{array}{l}
N \\
k
\end{array}\right) P_{e, k}\left(1-P_{r, e}\right)^{k}\left(P_{r, e}\right)^{N-k} \\
& \leq \sum_{k=0}^{N}\left(\begin{array}{l}
N \\
k
\end{array}\right) P_{e, k}\left(P_{r, e}\right)^{N-k} \\
& \leq M^{K}\left(4 N+8 M^{2 K}\right)^{N}\left(P d_{\text {min }}^{2}\right)^{-N} .
\end{aligned}
$$

Therefore, the diversity order of PDF II is $N$.

The analysis of SNR gain can be refined with a more careful analysis that tightens the probability bound in (51). The simulation results in Section VII show that PDF II has a significant performance gain over the other protocols.

\section{MAC Gain}

In this subsection, we note that variants of the protocols using two time slots have benefits at MAC layer when no central controller exists in the network and time division multiplexing is not employed.

Consider a network consisting of $N+2$ nodes without outside interference. In the four-time-slot protocols, the two terminals need to coordinate to determine which one should transmit in a given time slot. If we apply slotted ALOHA and assume that each terminal transmits with probability $p$ in a given time slot, the probability that one terminal transmits signal successfully to the relay nodes is $2 p(1-p)$, which is maximized when $p=1 / 2$. Hence, on average, one packet can be transmitted using fourtime-slot protocols, while two packets can be transmitted using two-time-slot protocols. Thus, two-time-slot protocols attain a $50 \%$ transmission saving at MAC layer.

\section{Simulation RESUlts}

We consider the average BER for two-relay networks averaged over the fading gains, ignoring direct transmission between the two terminals. If the constellation size used in two-

$$
\operatorname{det}^{-1}\left(\mathbf{I}_{2}+\frac{P}{16} \mathbf{M}\right)=\frac{1}{\left(1+\frac{P}{16}\left\|\mathbf{A}_{1} \boldsymbol{\Delta}_{1}\right\|_{2}^{2}\right)\left(1+\frac{P}{16}\left\|\mathbf{A}_{2} \boldsymbol{\Delta}_{2}\right\|_{2}^{2}\right)-\left\|\frac{P}{16} \mathbf{\Delta}_{1}^{H} \mathbf{A}_{1}^{H} \mathbf{A}_{2} \mathbf{\Delta}_{2}\right\|_{2}^{2}}
$$




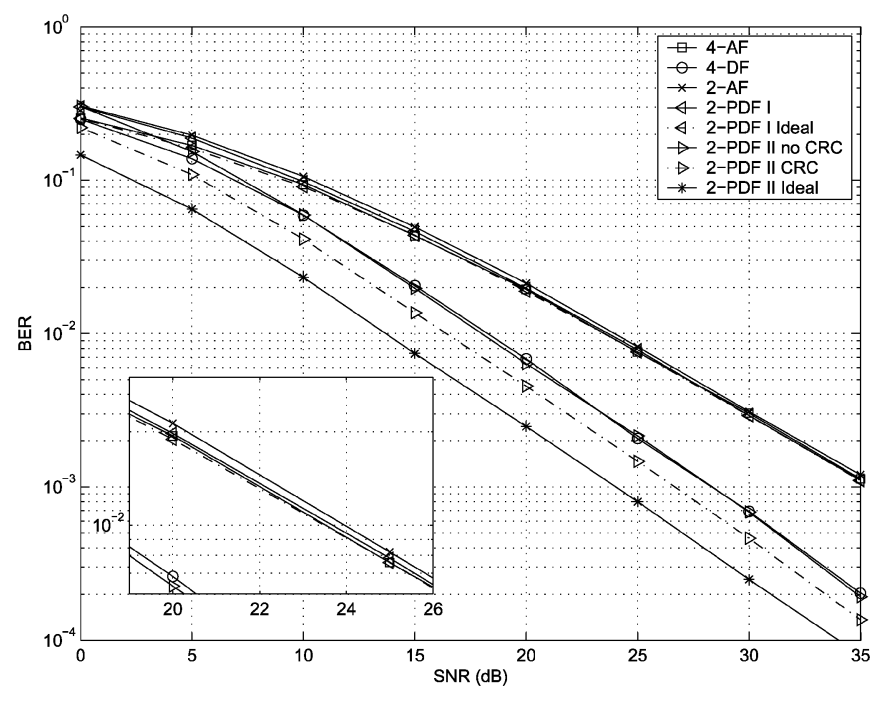

Fig. 2. Performance comparison of two-time-slot protocols and four-time-slot protocols in a network with a single relay. two-time-slot protocols use BPSK and four-time-slot protocols use 4-QAM.

time-slot protocols is $2^{B}$, then the constellation size used in four, three-time-slot protocols is $2^{2 B}$ and $2^{1.5 B}$, respectively, to equalize the data rate in all protocols. The rate reduction of PDF II due to the use of CRC is neglected. The channel coefficients $f_{i} \sim \mathcal{C N}(0,1)$ and $g_{i} \sim \mathcal{C N}(0,1), \forall i$ are used. Unless otherwise mentioned, random unitary matrices are used for LD code.

\section{A. No Direct Transmission Between Terminals}

1) Symmetric Networks: We first consider a symmetric network, where $f_{i} \sim \mathcal{C N}(0,1)$ and $g_{i} \sim \mathcal{C N}(0,1), \forall i$. We choose $P_{1}=P_{2}=P_{3}=0.5$.

Fig. 2 compares the performance of two-time-slot protocols and four-time-slot protocols in a network with a single relay. Two-time-slot protocols use BPSK and four-time-slot protocols use 4-QAM. The protocol $A$ using the orthogonal LD code (38) is denoted as $A_{o}$. PDF I and PDF II with no error on the uplink are used as benchmarks and are denoted as PDF ideal. PDF II without using CRC (PDF II no CRC) is also compared. In PDF II, by using CRC, the relay transmits 0 when it detects an error in the modular sum and in this case both terminals decodes +1 in the BPSK constellation. 4-AF has a $0.3-\mathrm{dB}$ gain over 2-AF at $\mathrm{BER}=5 \times 10^{-3}$. This result agrees with Theorem 3 when $N=1$. 4-DF performs very close to PDF II without CRC. PDF I performs slightly better than 2-AF, and it also performs close to PDF I Ideal. When CRC is applied, PDF II CRC has a $1.5-\mathrm{dB}$ gain over PDF II without CRC at BER $=5 \times 10^{-3}$. But PDF II $\mathrm{CRC}$ has a $1.5-\mathrm{dB}$ loss over PDF II Ideal at BER $=5 \times 10^{-3}$.

Fig. 3 compares the two-time-slot protocols using BPSK with four-time-slot protocols using 4-QAM with two relays. 4-AF has only a $0.3-\mathrm{dB}$ loss over $2-\mathrm{AF}$ at $\mathrm{BER}=2 \times 10^{-4}$. The loss predicted by Theorem 3 is $3-\mathrm{dB}$, which may come from the fact that we use union bound and other approximations in Theorem 3. 4-DF performs better than 4-AF, 2-AF, and PDF I in the observed low SNR. But 4-DF cannot achieve the diversity order 2. PDF I has a $0.5-\mathrm{dB}$ gain over $2-\mathrm{AF}$ at $\mathrm{BER}=2 \times 10^{-4}$. It seems that as the number of relays increases, PDF I will achieve

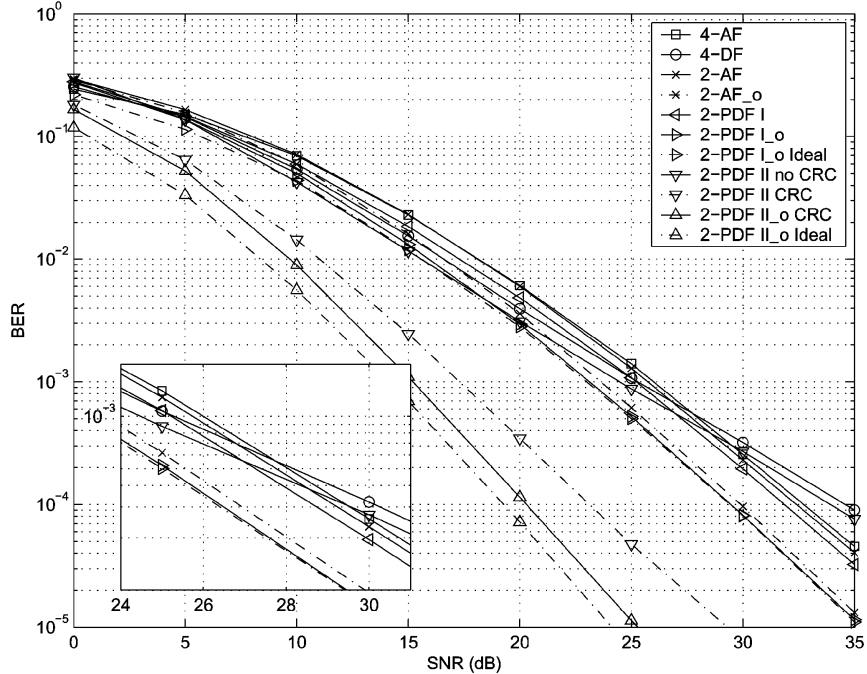

Fig. 3. Performance comparison of two-time-slot protocols and four-time-slot protocols in a network with two relays. two-time-slot protocols use BPSK and four-time-slot protocols use 4-QAM.

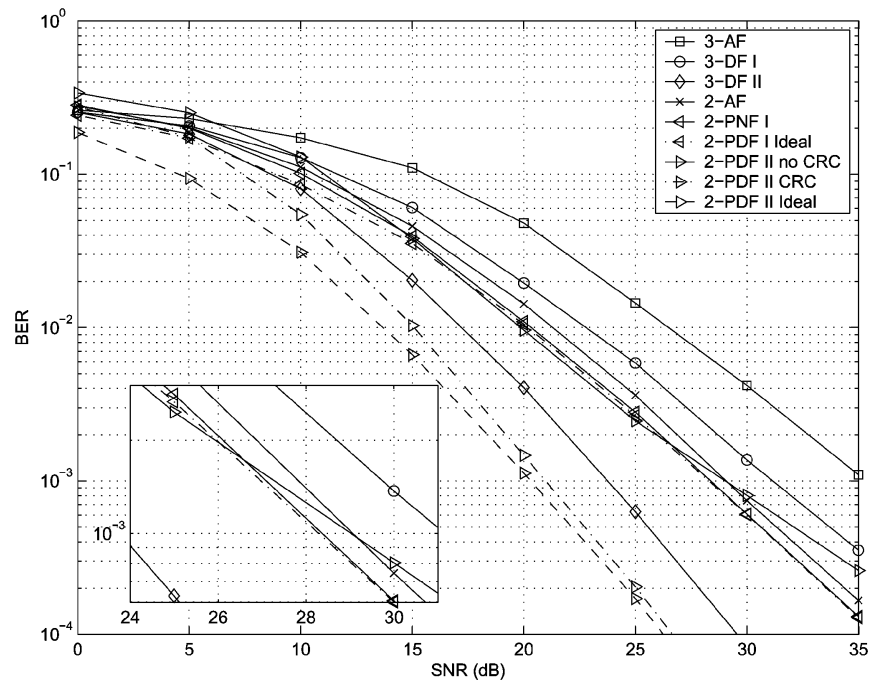

Fig. 4. Performance comparison of two-time-slot protocols and three-time-slot protocols in a network with two relays. two-time-slot protocols use 4-QAM and three-time-slot protocols use 8-QAM.

a higher gain over 2-AF. When the orthogonal code (38) is used, both 2-AF_o and PDF I_o can attain an additional 2.5-dB gain over those using random unitary matrices at $\mathrm{BER}=2 \times 10^{-4}$. In high SNR, PDF I_o achieves almost the same performance as PDF I_o Ideal, which means denoising is actually effective. PDF II CRC has a 8.3-dB gain over PDF II at BER $=2 \times 10^{-4}$. When orthogonal code is used, another 3-dB gain can be realized. PDF II_o CRC has a 1-dB loss over PDF II_o Ideal. However, when no CRC is used in PDF II, it can only achieve a diversity order one. But PDF II without CRC performs better than 4-DF. All these show that PDF II with CRC is a promising candidate for two-way relay networks. The gain attained by PDF II is from information embedding by using modular operation, together with the use of CRC.

Fig. 4 compares the performance of two-time-slot protocols using 4-QAM, and three-time-slot protocols using 8-QAM with 


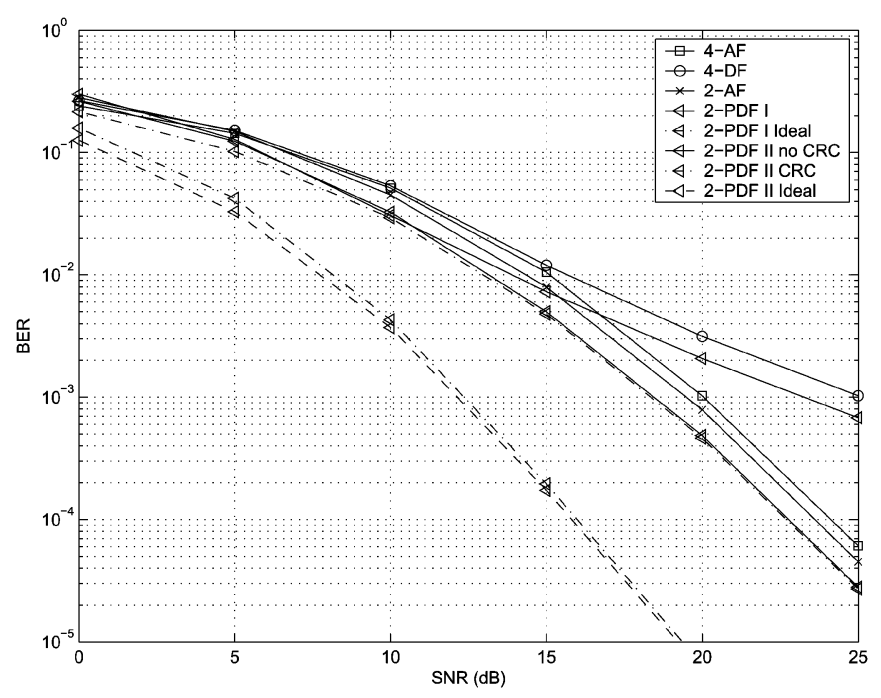

Fig. 5. Performance comparison of two-time-slot protocols and four-time-slot protocols in a network with four relays. two-time-slot protocols use BPSK and four-time-slot protocols use 4-QAM.

two relays. Due to the noise amplification, 3-AF performs worse than the other protocols. DF I has a 2.5-dB loss over PDF I and DF II has a 2.6-dB loss over PDF II at BER $=2 \times 10^{-3}$. This shows that when the constellation size increases while keeping the rate constant, two-time-slot protocols perform better. PDF I has a $0.7-\mathrm{dB}$ gain over $2-\mathrm{AF}$ at $\mathrm{BER}=2 \times 10^{-3}$. It seems that as the constellation size increases PDF I attains a larger gain over 2-AF. PDF II has a 7.3-dB gain over PDF I at BER $=10^{-3}$. PDF II has a 0.7-dB loss over PDF II Ideal at BER $=10^{-3}$.

Fig. 5 compares the performance of two-time-slot protocols using BPSK, and four-time-slot protocols using 4-QAM with four relays. Similar phenomenon is observed as with 2 relays. Both 4-DF and PDF II no CRC can only achieve a diversity order one. All other protocols seem to achieve a diversity order 4. By increasing the number of relays from 2 to 4 , the performance gain by using PDF II with CRC over that by using PDF I increases from 6.3 to $6.6 \mathrm{~dB}$ at $\mathrm{BER}=10^{-4}$, while the performance gap between 4-AF and 2-AF increases from 0.32 to $0.5 \mathrm{~dB}$ at $\mathrm{BER}=10^{-4}$.

In symmetric networks, these results suggest that for an average power constraint, the gain from using two-time-slot protocols over four-time-slot protocols increases by increasing the number of relays or increasing the constellation size when all protocols are of the same rate. Also, the gain of PDF I over 2-AF increases when the number of relays increases or the constellation size increases.

2) Asymmetric Networks: Finally, we consider an asymmetric network where the terminals are put $2 \mathrm{~m}$ apart. We assume that $f_{i} \sim \mathcal{C N}\left(0, \sigma^{2}\right)$ and $g_{i} \sim \mathcal{C N}\left(0, \eta^{2}\right)$, and $\sigma=1 / d, \eta=1 /(2-d)$, where $0 \leq d \leq 2$ is the distance from the relays to $\mathbb{T}_{1}$. Other parameters are the same as in Section VII-A-1). Due to symmetry, in Fig. 7, we only show the performance of different protocols as a function of the distance to terminal $\mathbb{T}_{1}$ at $\mathrm{SNR}=25 \mathrm{~dB}$. There are two relays in the network. Two-time-slot protocols use BPSK and four-time-slot protocols use 4-QAM. In AF protocols, the BER of signal from

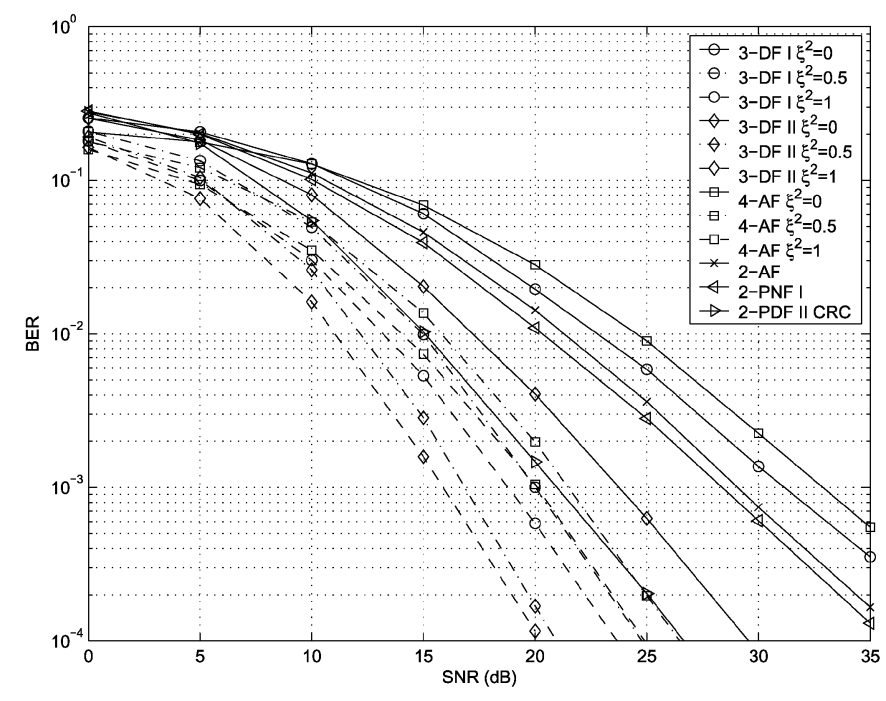

Fig. 6. Performance comparison of 2, 3, and four-time-slot protocols in a network with two relays when there is direct transmission between the two terminals. two-time-slot protocols use 4-QAM, three-time-slot protocols use 8-QAM, and four-time-slot protocols use 16-QAM.

$\mathbb{T}_{1}$ is better than that of $\mathbb{T}_{2}$. In this case, the channel from $\mathbb{T}_{1}$ to relays is stronger than that from $\mathbb{T}_{2}$ to relays. Therefore, the signal from $\mathbb{T}_{1}$ suffers from less noise amplification than that from $\mathbb{T}_{2}$. PDF I performs better than 2 -AF only when the relays lie around the midpoint between the two terminals. While there is a big performance difference between the two terminals by using PDF II, PDF II achieves better performance than all other protocols in the observed region. Different from AF protocols, the BER of signal from $\mathbb{T}_{2}$ is better than that of $\mathbb{T}_{1}$. This is because CRC is applied at the relays and the performance is affected only by the channel from the relays to the terminal. This result suggests the geometry of the network or whether channel gains on both sides of relays are balanced has different impact on different protocols. In practical networks where channel gains are generally unbalanced, choosing the best protocol requires careful consideration.

\section{B. Direct Transmission Between Terminals}

Let $d \sim \mathcal{C N}\left(0, \xi^{2}\right)$ denote the channel gain between the two terminals. We consider a network with two relays $(N=$ 2 ). Other parameters are the same as in Section VII-A-1). The signal is decoded using (31) at the two terminals.

From Fig. 6, with the increase of the strength of the direct channel $\xi^{2}$, the performance of the three-time-slot protocols improves. The diversity order of both DF I and DF II increases when $\xi^{2}$ increases from 0 to 0.5 . When $\xi^{2}$ further increases to 1 , DF I has a 1.2-dB gain over that with $\xi^{2}=0.5$ at $\mathrm{BER}=10^{-3}$, and DF II has a 1.2-dB gain over its counterpart. Both DF I and DF II perform better than 2-PDF II when $\xi^{2} \geq 0.5$. This suggests that the three-time-slot protocols are favorable when there is direct transmission between the two terminals. The performance limitation of two-time-slot protocols is due to the half-duplex constraint. When nodes in a networks operate in full-duplex mode, it is expected that simultaneous uplink transmission from the source terminals to the relay are still preferred. 


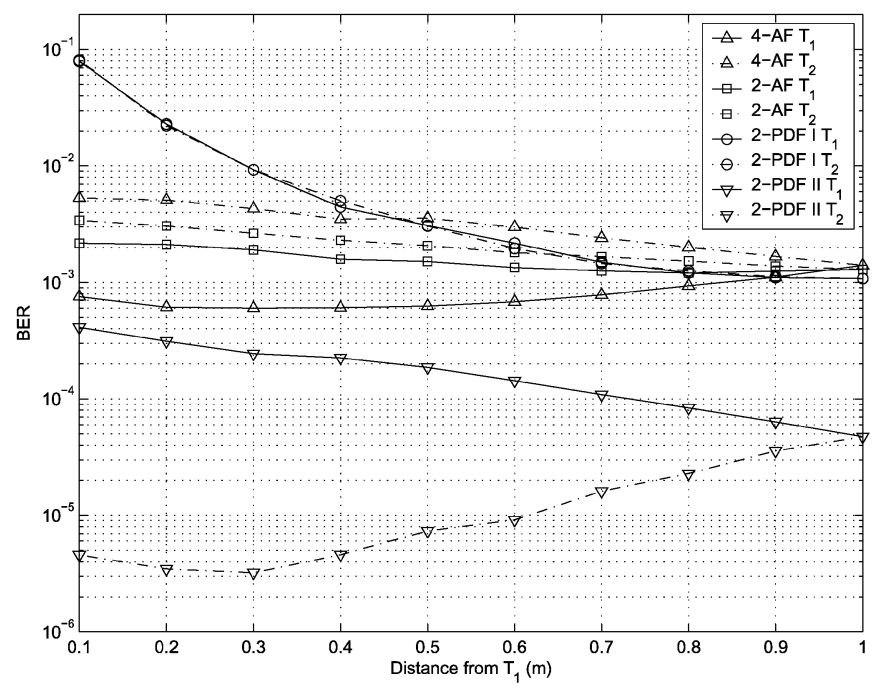

Fig. 7. Performance comparison of different protocols at $\mathrm{SNR}=25 \mathrm{~dB}$ as a function of the distance to terminal $\mathbb{T}_{1}$ in a network with two relays. two-timeslot protocols use BPSK and four-time-slot protocols use 4-QAM.

\section{CONCLUSION}

We have studied the use of LD space-time codes in two-way wireless relay networks. We proposed two new two-time-slot protocols, PDF I and PDF II, which can be considered as using network coding at the physical layer. When random unitary LD code is used, the PDF protocols are similar to random linear network coding [11], except that PDF operates on unitary group while random network coding operates over a finite field. To exploit the direct link between the two terminals, protocols using 3 time slots were also proposed and optimized. We have shown that the proposed AF protocols achieve the diversity order $\min \{N, K\}(1-(\log \log P / \log P))$, while PDF II achieves diversity order $N$. Furthermore, the two-time-slot protocols have MAC gain over the four-time-slot protocols.

\section{ACKNOWLEDGMENT}

The authors would like to thank the anonymous reviewers whose detailed comments have greatly improved the presentation of this paper.

\section{REFERENCES}

[1] J. Laneman, D. Tse, and G. Wornell, "Cooperative diversity in wireless networks: Efficient protocols and outage behavior," IEEE Trans. Inf. Theory, vol. 50, pp. 3062-3080, Dec. 2004.

[2] Y. Jing and B. Hassibi, "Distributed space-time coding in wireless relay networks," IEEE Trans. Wireless Commun., vol. 5, no. 12, pp. 3524-3536, Dec. 2006.

[3] S. Yiu, R. Schober, and L. Lampe, "Distributed space-time block coding," IEEE Trans. Commun., vol. 54, pp. 1195-1206, Jul. 2006.

[4] B. Mergen and A. Scaglione, "Randomized space-time coding for distributed cooperative communication," in Proc. IEEE ICC, Jun. 2006, pp. 4501-4506.

[5] T. Cover and A. Gamal, "Capacity theorems for the relay channel," IEEE Trans. Inf. Theory, vol. 25, pp. 572-584, Sep. 1979.

[6] C. E. Shannon, "Two-way communication channels," in Proc. 4th Berkeley Symp. Math. Stat. Prob., 1961, pp. 611-644.
[7] B. Rankov and A. Wittneben, "Spectral efficient signaling for half-duplex relay channels," in Proc. Asilomar Conf. Signals, Systems, Computers, Oct. 2005, pp. 1066-1071.

[8] B. Rankov and A. Wittneben, "Achievable rate regions for the two-way relay channel," in Proc. IEEE ISIT, Jul. 2006, pp. 1668-1672.

[9] C. Hausl and J. Hagenauer, "Iterative network and channel decoding for the two-way relay channel," in Proc. IEEE ICC, Jun. 2006, pp. $1568-1573$.

[10] R. Ahlswede, N. Cai, S. Y. R. Li, and R. W. Yeung, "Network information flow," IEEE Trans. Inf. Theory, vol. 46, pp. 1204-1216, Jul. 2000.

[11] T. Ho, M. Médard, R. Koetter, D. Karger, M. Effros, J. Shi, and B. Leong, "A random linear network coding approach to multicast," IEEE Trans. Inf. Theory, vol. 52, pp. 4413-4430, Oct. 2006.

[12] B. Hassibi and B. Hochwald, "High-rate codes that are linear in space and time," IEEE Trans. Inf. Theory, vol. 48, pp. 1804-1824, Jul. 2002.

[13] T. Cui, F. Gao, and A. Nallanathan, "Optimal training design for channel estimation in amplify and forward relay networks," in Proc. IEEE GLOBECOM, Nov. 2007, pp. 4015-4019.

[14] E. Viterbo and J. Bouros, "A universal lattice code decoder for fading channels," IEEE Trans. Inf. Theory, vol. 45, pp. 1639-1642, Jul. 1999.

[15] T. Cui and C. Tellambura, "Generalized feedback detection for spatial multiplexing multi-antenna systems," IEEE Trans. Wireless Commun., vol. 7, no. 2, pp. 594-603, Feb. 2008.

[16] T. Cui and C. Tellambura, "An efficient generalized sphere decoder for rank-deficient MIMO systems," IEEE Commun. Lett., vol. 9, pp. 423-425, May 2005.

[17] J. Laneman and G. Wornell, "Energy-efficient antenna sharing and relaying for wireless networks," in Proc. IEEE WCNC, 2000, pp. 7-12.

[18] T. Cui, T. Ho, and J. Kliewer, "Relay strategies for memoryless two-way relay channels: Performance analysis and optimization," in Proc. IEEE ICC, May 2008, pp. 1139-1143.

[19] Y. Jing and B. Hassibi, "Diversity analysis of distributed space-time codes in relay networks with multiple transmit/receive antennas," EURASIP J. Adv. Signal Process., vol. 8, no. 2, pp. 1-17, Jan. 2008.

[20] G. Golub and C. V. Loan, Matrix Computations, 3rd ed. Baltimore, MD: The Johns Hopkins Univ. Press, 1996.

[21] V. Tarokh, N. Seshadri, and A. Calderbank, "Space-time codes for high data rate wireless communication: Performance criterion and code construction," IEEE Trans. Inf. Theory, vol. 44, pp. 744-765, Mar. 1998.

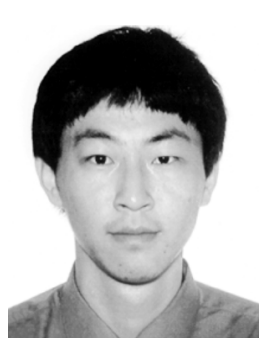

Tao Cui (S'04) received the M.Sc. degree in the Department of Electrical and Computer Engineering, University of Alberta, Edmonton, AB, Canada, in 2005 and the M.S. degree from the Department of Electrical Engineering, California Institute of Technology, Pasadena, in 2006. He is currently working toward the Ph.D. degree at the Department of Electrical Engineering, California Institute of Technology.

His research interests are in the interactions between networking theory, communication theory, and

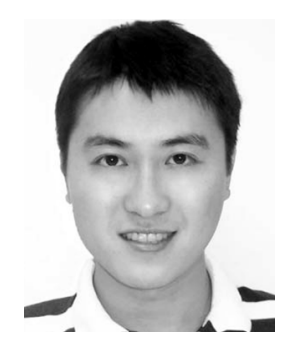

Feifei Gao (S'05-M'09) received the B.Eng. degree in information engineering from Xi' an Jiaotong University, Xi' an, Shaanxi China, in 2002, the M.Sc. degree from the McMaster University, Hamilton, ON, Canada in 2004, and the Ph.D. degree from the National University of Singapore in 2007.

$\mathrm{He}$ is currently working as a Research Fellow at Institute for Infocomm Research, A*STAR, Singapore. His research interests are in communication theory, broadband wireless communications, signal processing for communications, MIMO systems, and array signal processing.

Mr. Gao was a recipient of the president scholarship from the National University of Singapore. He has coauthored more than 40 refereed IEEE journal and conference papers and has served as a TPC member for IEEE ICC (2008, 2009), IEEE VTC (2008), and IEEE GLOBECOM (2008). 


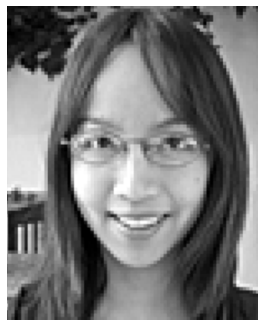

Tracey Ho (M'05) received the B.S. and M.Eng. degrees in 1999 and the Ph.D. degree in 2004, all in electrical engineering and computer science (EECS), from the Massachusetts Institute of Technology (MIT).

$\mathrm{He}$ is an Assistant Professor in electrical engineering and computer science at the California Institute of Technology, Pasadena. Her primary research interests are in information theory, network coding, and communication networks.

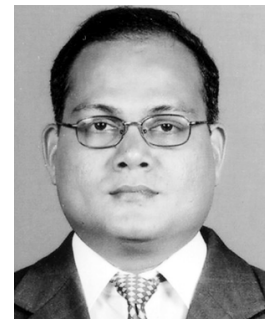

Arumugam Nallanathan (S'97-M'00-SM'05) received the B.Sc. degree with honors from the University of Peradeniya, Sri-Lanka, in 1991, the Certificate of Postgraduate Studies in Engineering from Cambridge University, Cambridge, U.K., in 1994, and the Ph.D. degree from the University of Hong Kong, Hong Kong, in 2000, all in electrical engineering.

He was an Assistant Professor in the Department of Electrical and Computer Engineering, National University of Singapore, Singapore, from August 2000 to December 2007. Currently, he is a Senior Lecturer in the Department of Electronic Engineering at King's College London, London, U.K. His research interests include cooperative communications, cognitive radio, MIMO-OFDM systems, ultra-wide bandwidth (UWB) communication, and localization. In these areas, he has published over 130 journal and conference papers.

Dr. Nallanathan is a corecipient of the Best Paper Award presented at the 2007 IEEE International Conference on Ultra-Wideband (ICUWB). He currently serves on the Editorial Board of the IEEE Transactions on Wireless Communications and IEEE Transactions on Vehicular Technology as an Associate Editor. He served as a Guest Editor for the EURASIP JOURNAL OF WIRELESS COMMUNICATIONS AND NETWORKING Special issue on UWB Communication Systems-Technology and Applications. He served as a Technical Program Committee Member for more than 30 IEEE international conferences. He also served as the General Track Chair for the IEEE VTC'2008-Spring. $\mathrm{He}$ currently serves as the Co-Chair for the IEEE GLOBECOM 2008 Signal Processing for Communications Symposium, and the IEEE ICC 2009 Wireless Communications Symposium. 